\title{
A GENERAL NOTION OF UNIFORM STRATEGIES
}

\author{
BASTIEN MAUBERT \\ Team LOGICA, IRISA, Université de Rennes 1 \\ Rennes, 35042, France \\ bastien.maubert@irisa.fr \\ SOPHIE PINCHINAT \\ Team LOGICA, IRISA, Université de Rennes 1 \\ Rennes, 35042, France \\ sophie.pinchinat@irisa.fr \\ Received (Day Month Year) \\ Revised (Day Month Year)
}

\begin{abstract}
We consider two-player turn-based game arenas for which we investigate uniformity properties of strategies. These properties involve sets of plays in order to express useful constraints on strategies that are not $\mu$-calculus definable. Typically, we can represent constraints on allowed strategies, such as being observation-based. We propose a formal language to specify uniformity properties, involving an original modality called $R$, meaning "for all related plays". Its semantics is given by some binary relation between plays. We demonstrate the relevance of our approach by rephrasing various known problems from the literature. We also study an automated procedure to synthesize strategies subject to a uniformity property, which strictly extends existing results based on, say standard temporal logics. We exhibit a generic solution for the synthesis problem provided the binary relation that defines the sets of related plays is recognizable by a finite state transducer. This solution yields a non-elementary procedure.
\end{abstract}

Keywords: Game Theory, Uniform Strategies, Epistemic Temporal Logic, Rational Relations

Subject Classification: 22E46, 53C35, 57S20.

\section{Introduction}

In extensive (finite or infinite duration) games, the arena is represented as a graph whose vertices denote positions of players and whose paths denote plays. In this context, a strategy of a player is a mapping prescribing to this player which next position to select provided she has to make a choice at this current point of the play. As mathematical objects, strategies can be seen as infinite trees those of which are obtained by pruning the infinite unfolding of the arena according to the selection prescribed by this strategy; outcomes of a strategy are therefore the branches of this tree.

Strategies of players are not arbitrary in general, since players aim at achieving some objectives: in classic game theory with finite-duration plays, the reasonable 
rationality assumption leads players to play in such a way that they maximize their pay-off. More recently, infinite-duration game models have been intensively studied for their applications in computer science (Apt and Grädel [2011]) and logic (Grädel et al. [2002], ). First, infinite-duration games provide a natural abstraction of computing systems' non-terminating interaction (Alur et al. [2002], ) (think of a communication protocol between a printer and its users, or control systems). Second, infinite-duration games naturally occur as a tool to handle logical systems for the specification of non-terminating behaviors, such as for the propositional $\mu$ calculus (Emerson and Jutla [1991]), leading to a powerful theory of automata, logics and infinite games (Grädel et al. [2002], ) and to the development of algorithms for the automatic verification ("model-checking") and synthesis of hardware and software systems. In both cases, outcomes of strategies are submitted to $\omega$-regular conditions representing some desirable property of a system.

Additionally, the cross fertilization of multi-agent systems and distributed systems theories has led to equip logical systems with additional modalities, such as epistemic ones, to capture uncertainty (Sato [1977]; Lehmann [1984]; Fagin et al. [1991]; Parikh and Ramanujam [1985]; Ladner and Reif [1986]; Halpern and Vardi [1989], ), and more recently, these logical systems have been adapted to game models in order to reason about knowledge, time and strategies (van der Hoek and Wooldridge [2003]; Jamroga and van der Hoek [2004]; Dima et al. [2010], ). The whole picture then becomes intricate, mainly because time and knowledge are essentially orthogonal, yielding a complex theoretical universe to reason about. In order to understand to which extent knowledge and time are orthogonal, the angle of view where strategies are infinite trees is helpful: Time is about the vertical dimension of the trees as it relates to the ordering of encountered positions along plays (branches) and to the branching in the tree. On the contrary, Knowledge is about the horizontal dimension, as it relates plays carrying, e.g., the same information.

As far as we know, this horizontal dimension, although extensively studied when interpreted as knowledge or observation (Arnold et al. [2003]; van der Hoek and Wooldridge [2003]; Jamroga and van der Hoek [2004]; Van Benthem [2005]; Pinchinat and Riedweg [2005]; Chatterjee et al. [2006]; Alur et al. [2007]; Dima et al. [2010], ), has not been addressed in its generality. In this paper, we aim at providing a unified setting to handle it. We introduce the generic notion of uniformity properties and associated so-called uniform strategies (those satisfying uniformity properties). Some notions of uniform strategies have already been defined in the literature. The most famous one concerns games with imperfect information, in which the players have some uncertainty concerning the current configuration of the game (think of Poker for example). Naturally, a player in such a game cannot plan to play differently in situations that she cannot distinguish: her strategies must be defined uniformly over observationally equivalent situations, reason why strategies for games with imperfect information have sometimes been called uniform strategies (Van Benthem [2001]; Van Benthem [2005]; 
Jamroga and van der Hoek [2004]). Another notion of uniform strategy has been used to define the evaluation game of Dependence Logic (Väänänen [2007]), and this notion, though different from the one of imperfect-information games, also consists in a constraint on sets of somehow related outcomes. Both these notions of uniform strategies fall into the general framework we present here.

We have chosen to tell our story in a simple framework where games are described by two-player turn-based arenas in which all information is put inside the positions, and not on the edges. However, the entire theory can be adapted to more sophisticated models, e.g. with labels on edges, multi-players, concurrent games ... Additionally, although uniformity properties can be described in a set-theoretic framework, we have chosen to use a logical formalism which can be exploited to address fundamental automated techniques such as the verification of uniformity properties and the synthesis of uniform strategies - arbitrary uniformity properties are in general hopeless for automation. The formalism we use combines the Lineartime Temporal Logic LTL (Gabbay et al. [1980], ) and a new modality $R$ (for "for all related plays"), the semantics of which is given by a binary relation between plays. Modality $R$ generalizes the knowledge operator $K$ of (Halpern and Vardi [1989]) for the epistemic relations of agents in Interpreted Systems. The semantic binary relations between plays are very little constrained: they are not necessarily equivalences, to capture, e.g. plausibility (pre)orders one finds in doxastic logic (Hintikka [1962]), neither are they knowledge-based, to capture particular strategies in games where epistemic aspects are irrelevant. Formulas of the logic are interpreted over outcomes of a strategy. The $R$ modality allows to universally quantify over all plays that are in relation with the current play. Distinguishing between the universal quantification over all related plays in the game and the universal quantification over only those related plays that also are outcomes of the strategy yields two kinds of uniform strategies: the fully-uniform strategies and the strictly-uniform strategies.

We illustrate the suitability of our notions by borrowing many frameworks from the literature: strategies for games with imperfect information, games with opacity condition, the non-interference properties of computing systems, diagnosability of discrete-event systems (with a proposal for a formal definition of prognosis), and finally the evaluation game for Dependence Logic. Proofs of Section 3 are omitted due to lack of space, but they are quite simple. Through these examples we show that both notions, strict uniformity and full uniformity, are relevant and incomparable. These examples also demonstrate that defining uniformity properties with our formal language is convenient and intuitive. There are even more instances of uniform strategies in the literature, but the numerous examples we give here are already convincing enough to justify the relevance of the notion.

Next we turn to the automated synthesis of uniform strategies. For this purpose, we unsurprisingly restrict to finite arenas and to binary relations between plays that are finitely representable: we use finite state transducers (Berstel [1979]), an adequate device to characterize a large class of binary relations between sequences of 
symbols, hence they can be used to relate sequences of positions, i.e. plays. Incidentally, all binary relations that are involved in the relevant literature seem to follow this restriction. In this context, we address the problem of the existence of a fully-uniform strategy: "given a finite arena, a finite state transducer describing a binary relation between plays, and a formula expressing a uniformity property, does there exist a fully-uniform strategy for Player 1?". We prove that this problem is decidable by designing an algorithm. This algorithm involves a non-trivial powerset construction from the arena and the finite state transducer. This construction needs being iterated a number of times that matches the maximum number of nested $R$ modalities in the formula specifying the uniformity property. As each powerset construction is computed in exponential time, the overall procedure is non-elementary. Regarding the decision problem for the existence of a strictly-uniform strategy, its decidability is an open problem.

The paper is organized as follows. in Section 2 we set the mathematical framework: we introduce the notion of uniform strategies and we present the formal language to specify uniformity properties. Next, in Section 3, we expose a significant set of six instances of uniform strategy problems from the literature. Section 4 is dedicated to a short reminder about finite state transducers that are used in the strategy synthesis problem addressed and solved in Section 5. We finish by a discussion on the work done and perspectives in Section 6 .

\section{Uniform strategies}

In this section we define a very general notion of uniform strategies. We consider two-player turn-based games that are played on graphs with vertices labelled with propositions. These propositions represent the relevant information for the uniformity properties one wants to state. If the game models a dynamic system interacting with its environment, relevant information can be the value of some (Boolean) variables. If it models agents interacting in a network, interesting information can be the state of the communication channels. In games with imperfect information, it can be what action has just been played.

From now on and for the rest of the paper, we let $A P$ be an infinite set of atomic propositions.

An arena is a structure $\mathcal{G}=\left(V, E, v_{I}, \ell\right)$ where $V=V_{1} \uplus V_{2}$ is the set of positions, partitioned between positions of Player $1\left(V_{1}\right)$ and those of Player $2\left(V_{2}\right)$, $E \subseteq\left(V_{1} \times V_{2}\right) \cup\left(V_{2} \times V_{1}\right)$ is the set of edges, $v_{I} \in V$ is the initial position and $\ell: V \rightarrow \mathcal{P}(A P)$ is a valuation function, mapping each position to the finite set of atomic propositions that hold in this position.

For $v \in V$, $\operatorname{Traces}_{\omega}(v) \subseteq v V^{\omega}$ is the set of infinite traces starting in $v$, i.e. the set of infinite paths $v_{0} v_{1} v_{2} \ldots$ in the game graph $(V, E)$, with $v_{0}=v$, and similarly Traces $_{*}(v) \subseteq v V^{*}$ is the set of finite traces starting in $v$, i.e. the set of finite paths $v_{0} v_{1} \ldots v_{n}$ in $(V, E)$, with $v_{0}=v$ and $n \geq 0$. We let $\operatorname{Traces}_{\omega}=\cup_{v \in V} \operatorname{Traces}_{\omega}(v)$ and $\operatorname{Traces}_{*}=\cup_{v \in V} \operatorname{Traces}_{*}(v)$. Typical elements of $\operatorname{Traces}_{\omega}$ are $\pi, \pi^{\prime}$, and $\lambda, \lambda^{\prime}$ 
are typical elements of Traces $_{*}$. Plays $\omega$ denotes Traces $_{\omega}\left(v_{I}\right)$ and Plays $s_{*}$ denotes Traces $_{*}\left(v_{I}\right)$, respectively the set of infinite and finite plays in the game. We shall write $\rho$ instead of $\lambda$ to distinguish finite plays from other finite traces.

For an infinite trace $\pi=v_{0} v_{1} \ldots$ and $i, j \in \mathbb{N}, \pi[i]:=v_{i}, \pi[i, \infty]:=v_{i} v_{i+1} \ldots \in$ Traces $_{\omega}$ and $\pi[i, j]:=v_{i} \ldots v_{j} \in$ Traces $_{*}$. We will use similar notations for finite traces, and |.| : Traces $_{*} \cup$ Traces $_{\omega} \rightarrow \mathbb{N} \cup\{\omega\}$ denotes the length of the trace. If $\lambda \in$ Traces $_{*}$, we let last $(\lambda):=\lambda[|\lambda|-1]$ be the last position of $\lambda$.

A strategy for Player $k, k \in\{1,2\}$, is a partial function $\sigma:$ Plays $_{*} \rightarrow V$ that assigns the next position to choose in every situation in which it is Player $k$ 's turn to play. In other words $\sigma(\rho)$ is defined if last $(\rho) \in V_{k}$. Let $\sigma$ be a strategy for Player $k$. We say that a play $\pi \in$ Plays $_{\omega}$ is induced by $\sigma$ if for all $i \geq 0$ such that $\pi[i] \in V_{k}$, $\pi[i+1]=\sigma(\pi[0, i])$, and the outcome of $\sigma$, noted $\operatorname{Out}(\sigma) \subseteq$ Plays $_{\omega}$, is the set of all infinite plays that are induced by $\sigma$.

We want to express properties of strategies that do not concern only single traces but rather sets of correlated traces. We first give a very abstract definition.

Definition 1. Let $\mathcal{G}$ be an arena. A uniformity property $U \subseteq \mathcal{P}\left(\right.$ Plays $\left._{\omega}\right)$ is a set of sets of plays in $\mathcal{G}$.

Definition 2. Let $\mathcal{G}$ be an arena and $U$ a uniformity property. A strategy $\sigma$ is $U$-uniform if $\operatorname{Out}(\sigma) \in U$.

This definition gives an idea of the notion we want to capture, but this settheoretic definition is not very intuitive, and moreover it is so expressive that automatically handling this notion in its generality is hopeless. We therefore restrict the notion of uniform strategy by fixing a formal language to specify uniformity properties. As demonstrated in the next section, the language is powerful enough to capture plethora of instances from the literature.

The proposed language enables to express properties of the dynamics of plays, and resembles the Linear Temporal Logic (LTL) (Gabbay et al. [1980], ). However, while LTL formulas are evaluated on individual plays (paths), we want here to express properties on sets of plays. To this aim, we equip arenas with a binary relation between finite plays, and we enrich the logic with a modality $R$ that quantifies over related plays: the intended meaning of $R \varphi$ is that $\varphi$ holds in every related play.

For the general presentation of the logic, we do not make yet assumptions concerning the binary relation over plays, as opposed to Section 5 dedicated to decidability issues.

We now give the syntax and semantics of the language $\mathcal{L}$.

\subsection{Syntax}

The syntax of the language $\mathcal{L}$ is the following :

$$
\mathcal{L}: \varphi, \psi::=p|\neg \varphi| \varphi \wedge \psi|\bigcirc \varphi| \varphi \mathcal{U} \psi \mid R \varphi
$$


where $p$ is in $A P$. As usual we will use the following notations : true $:=p \vee \neg p$, false $:=\neg$ true, $\mathbf{F} \varphi:=$ true $\mathcal{U} \varphi, \mathbf{G} \varphi:=\neg \mathbf{F} \neg \varphi$, and $\varphi \mathcal{W} \psi:=\varphi \mathcal{U} \psi \vee \mathbf{G} \varphi$. In addition we will use the following notation: $\langle R\rangle \varphi:=\neg R \neg \varphi$, and for a formula $\varphi \in \mathcal{L}, \operatorname{Sub}(\varphi)$ denotes the set of all its subformulas.

The syntax of $\mathcal{L}$ is similar to that of linear temporal logic with knowledge (Halpern and Vardi [1989]). However, we use $R$ instead of the usual knowledge operator $K$ to emphasize that though it has a strong epistemic flavour, notably in various application instances we present here, it need not be interpreted in terms of knowledge in general, but merely as a way to state properties of sets of plays.

Definition 3. For a formula $\varphi \in \mathcal{L}$, we define the $R$-depth of $\varphi$, denoted $d_{R}(\varphi)$, as the maximum number of nested $R$ modalities in $\varphi$. For $n \geq 0$, let $\mathcal{L}_{n}=\{\varphi \in \mathcal{L} \mid$ $\left.d_{R}(\varphi)=n\right\}$ be the set of formulas of $R$-depth $n$.

We note LTL the language $\mathcal{L}_{0}$ as it matches the syntax (and also the semantics) of the Linear-time Temporal Logic.

\subsection{Semantics}

Take an arena $\mathcal{G}=\left(V, E, v_{I}, \ell\right)$ and a relation $\sim \subseteq$ Plays $_{*} \times$ Plays $_{*}$. A formula $\varphi$ of $\mathcal{L}$ is evaluated at some point $i \in \mathbb{N}$ of a play $\pi \in$ Plays $_{\omega}$, within a universe $\Pi \subseteq$ Plays $_{\omega}$. The semantics is given by induction over formulas.

$\Pi, \pi, i=p \quad$ if $p \in \ell(\pi[i])$

$\Pi, \pi, i \models \neg \varphi \quad$ if $\Pi, \pi, i \not \models \varphi$

$\Pi, \pi, i \models \varphi \wedge \psi \quad$ if $\Pi, \pi, i \models \varphi$ and $\Pi, \pi, i \models \psi$

$\Pi, \pi, i \models \bigcirc \varphi \quad$ if $\Pi, \pi, i+1 \models \varphi$

$\Pi, \pi, i=\varphi \mathcal{U} \psi$ if there is $j \geq i$ s.t. $\Pi, \pi, j \models \psi$ and for all $i \leq k<j, \Pi, \pi, k=\varphi$

$\Pi, \pi, i \models R \varphi \quad$ if for all $\pi^{\prime} \in \Pi, j \in \mathbb{N}$ s.t. $\pi[0, i] \sim \pi^{\prime}[0, j], \Pi, \pi^{\prime}, j \models \varphi$

The LTL part is classic. $R \varphi$ is true at some point of a play if $\varphi$ is true in every related finite play in the universe.

We will sometimes need to evaluate an LTL-formula $\varphi$ in a position $v$ of an arena, with the classic semantics that $\varphi$ holds in $v$ if it holds in every trace starting in $v$. Formally, for an arena $\mathcal{G}=\left(V, E, v_{I}, \ell\right)$, a position $v \in V$ and a formula $\varphi \in$ LTL, we write

$$
\mathcal{G}, v \models \varphi \text { if } \pi, 0 \models \varphi \text { for all } \pi \in \operatorname{Traces}_{\omega}(v)
$$

Here we can omit $\Pi$ because the formula has no $R$ modality.

Definition 4. Given an arena $\mathcal{G}=\left(V, E, v_{I}, \ell\right)$, a uniformity property is a pair $(\sim, \varphi)$ where $\sim$ is a binary relation over Plays $*$ and $\varphi \in \mathcal{L}$ is a formula.

Now we define two notions of uniform strategies, which differ only in the universe the $R$ modality quantifies over: Out $(\sigma)$ or Plays $_{\omega}$ (with the latter, related plays not induced by the strategy also count). As we shall see in the examples of the next section, making a nuance is worthwhile. 
Definition 5. Let $\mathcal{G}$ be an arena and $(\sim, \varphi)$ be a uniformity property. A strategy $\sigma$ is

- $(\sim, \varphi)$-strictly-uniform if for all $\pi \in \operatorname{Out}(\sigma), \operatorname{Out}(\sigma), \pi, 0=\varphi$.

- $(\sim, \varphi)$-fully-uniform if for all $\pi \in \operatorname{Out}(\sigma)$, Plays $_{*}, \pi, 0=\varphi$.

The notion of fully-uniform strategy is in a sense weaker than the strictlyuniform one. Indeed, the fact that a particular play in an arena verifies a formula $\varphi$ in the fully-uniform semantics, i.e. with Plays ${ }_{\omega}$ and not $\operatorname{Out}(\sigma)$ as a universe, is independent of the strategy considered. Hence in the general definition of uniform strategies, a uniformity property $U$ could be defined to be a set of plays instead of a set of sets of plays, and a strategy $\sigma$ could be said to be uniform if $\operatorname{Out}(\sigma) \subseteq U$ instead of $\operatorname{Out}(\sigma) \in U$, it would still contain the notion of fully-uniform strategies. Strictly-uniform strategies could no longer fit in this definition though, as deciding whether a play verifies a formula in this semantics cannot be done without knowing the strategy. In this sense, strict uniformity is "stronger" than full uniformity. However, the notion of fully-uniform strategies still enables to characterize tree languages that are not even $\mu$-calculus definable: indeed, as observed by Alur et al. [2006], , given a infinite tree, Property $\left(^{*}\right)$ that at every depth $d>0$, there exist two nodes, one of which being labeled by, say $p$, and one of which not being labeled by $p$, cannot be $\omega$-regular (a pumping lemma argument suffices). Hence this property cannot be defined by any $\mu$-calculus formula. However, we are able to characterize arenas whose tree unfolding has this property: consider the equivalence relation $=$ length which relates plays with equal length. One easily sees that the existence of a (= length, $\mathbf{G}(\langle R\rangle p \wedge\langle R\rangle \neg p)$ )-fully-uniform strategy is equivalent to say that the tree unfolding of the arena has Property $(*)$.

Remark also that the relation $\sim$ plays no role in Definition 5 if $\varphi$ does not contain any $R$ operator, i.e. is a mere LTL formula. Notice that in this latter case, some standard $\omega$-regular (winning) conditions can be expressed over plays. The extension to a more powerful logic, such as the full propositional $\mu$-calculus, in order to capture all $\omega$-regular properties is a priori possible, even though the decision procedure of Section 5 seems to work only for the alternation-free fragment. In this work we choose to take LTL as base language as it is sufficient for the motivating examples considered in Section 3.

\section{Frameworks from the literature}

In this section we demonstrate that the notion of uniform strategy of previous Section 2 enables to embed various problems from the literature, and in particular that it subsumes two existing notions of uniform strategies.

\subsection{Games with imperfect information}

Games with imperfect information, in general, are games in which some of the players do not know exactly what is the current position of the game. This can occur in 
real games, e.g. poker since one does not know what cards her opponents have in hands, but also in situations arising from computer science, like for example a program that observes or controls a system by means of a sub-part of its variables, the interface, while other variables remain hidden. One important aspect of imperfectinformation games is that not every strategy is "playable". Indeed, a player who has imperfect information cannot plan to play differently in situations that she cannot distinguish. This is why strategies are required to choose moves uniformly over observationally equivalent situations. This kind of strategies is sometimes called uniform strategies in the community of strategic logics (Van Benthem [2001]; Van Benthem [2005]; Jamroga and van der Hoek [2004]), or observation-based strategies in the community of computer-science oriented game theory (Chatterjee et al. [2006], ). In fact, all the additional complexity of solving imperfect-information games, compared to perfect-information ones, lies in this constraint put on strategies.

In games with imperfect information, the player's ability to remember what happened so far along a play is a key point to achieve a winning strategy, as opposed to e.g. perfect-information parity games, where memoryless strategies are sufficient. Moreover in an imperfect information configuration, it is necessary to define what situations are indistinguishable to the player, and this requires defining how much memory she has of what occurs in a play. It is therefore relevant under an imperfect information assumption to distinguish the perfect recall setting, as opposed to the imperfect recall one. In the former, the player remembers the whole history of the observation she had of a play, no matter how long it is, while in the latter the player forgets a part of the information. An agent with imperfect recall can either be memoryless, i.e. he does not remember anything and takes his decisions only based on the current position, or have a bounded memory, but this case can be reduced to the memoryless case by putting the different possible configurations of his memory in the positions of the arena.

While games with imperfect information and perfect recall have been studied intensively (Reif [1984]; Chatterjee et al. [2006]; Berwanger and Doyen [2008], ), the case of imperfect recall has received much less attention since paradoxes concerning the interpretation of such games were raised (Piccione and Rubinstein [1997]). Nonetheless, relevant problems may be modeled with imperfect recall: typically, particular computing resources have very limited memory and cannot remember arbitrarily long histories.

In this subsection, we show that the notion of "uniform" or "observation-based" strategy, and hence the essence of games with imperfect information, can be easily embedded in our notion of uniform strategy, and this no matter the assumption made on the memory of the player.

We consider two-player imperfect-information games as studied for example in Reif [1984]; Chatterjee et al. [2006]; Berwanger and Doyen [2008], . In these games, Player 1 only partially observes the positions of the game, such that some positions are indistinguishable to her, while Player 2 has perfect information (the asymmetry 
is due to the focus being on the existence of strategies for Player 1). Arenas are labelled directed graphs together with a finite set of actions Act, and in each round, if the position is a node $v$, Player 1 chooses an available action $a$, and Player 2 chooses a next position $v^{\prime}$ reachable from $v$ through an $a$-labelled edge.

We equivalently define this framework in a manner that fits our setting by putting Player 1's actions inside the positions. We have two kinds of positions, of the form $v$ and of the form $(v, a)$. In a position $v$, when she chooses an action $a$, Player 1 actually moves to position $(v, a)$, then Player 2 moves from $(v, a)$ to some $v^{\prime}$. So an imperfect-information game arena is a structure $\mathcal{G}_{\text {imp }}=(\mathcal{G}, \sim)$ where $\mathcal{G}=\left(V, E, v_{I}, \ell\right)$ is a two-player game arena with positions in $V_{1}$ of the form $v$ and positions in $V_{2}$ of the form $(v, a)$. For a position $(v, a) \in V_{2}$, we note $(v, a)$.act $:=a$. $E \subseteq V_{1} \times V_{2} \cup V_{2} \times V_{1}, v E\left(v^{\prime}, a\right)$ implies $v=v^{\prime}, v_{I} \in V_{1}$. We assume that $p_{1} \in A P$ and for every action $a$ in $A c t,, p_{a} \in A P$. $p_{1}$ holds in positions belonging to Player 1, and $p_{a}$ holds in positions of Player 2 where the last action chosen by Player 1 is $a: \ell(v)=\left\{p_{1}\right\}$ for $v \in V_{1}, \ell(v, a)=\left\{p_{a}\right\}$ for $(v, a) \in V_{2}$. Finally, $\sim \subseteq V_{1}^{2}$ is an observational equivalence relation on positions, that relates indistinguishable positions for Player 1 . We define its extension to finite plays as the least relation $\approx$ such that $\rho \cdot v \approx \rho^{\prime} \cdot v^{\prime}$ whenever $\rho \approx \rho^{\prime}$ and $v \sim v^{\prime}$, and $\rho \cdot(v, a) \approx \rho^{\prime} \cdot\left(v^{\prime}, a^{\prime}\right)$ whenever $\rho \approx \rho^{\prime}, v \sim v^{\prime}$ and $a=a^{\prime}$.

We add the classic requirement that the same actions must be available in indistinguishable positions: for all $v, v^{\prime} \in V_{1}$, if $v \sim v^{\prime}$ then $v E(v, a)$ if, and only if, $v^{\prime} E\left(v^{\prime}, a\right)$. In other words, if a player has different options, she can distinguish the positions.

Definition 6. A strategy $\sigma$ for Player 1 is observation-based if for all $\rho, \rho^{\prime} \in$ $v\left(V_{2} V_{1}\right)^{*}, \rho \approx \rho^{\prime}$ implies $\sigma(\rho)$.act $=\sigma\left(\rho^{\prime}\right)$.act.

We define the formula

$$
\text { SameAct }:=\mathbf{G}\left(p_{1} \rightarrow \bigvee_{a \in A c t} R \bigcirc p_{a}\right)
$$

which expresses that whenever it is Player 1's turn to play, there is an action $a$ that is played in all equivalent finite plays.

Theorem 1. A strategy $\sigma$ for Player 1 is observation-based if, and only if, it is $(\approx$, SameAct)-strictly-uniform.

Here we have to make use of the notion of strict uniformity, and not the full uniformity. Indeed, after a finite play $\pi[0, i]$ ending in $V_{1}$, we want to enforce that the actions in the next positions of equivalent plays are the same only in those plays that are induced by the strategy we consider, and not in every possible play in the game. This would of course not hold as soon as several choices are possible in $\pi[i]$.

Also, it is interesting to see that this notion could also be embedded with a simpler formula by using a relation that is not an equivalence. Define $\sim$ as: $\pi[0, i] \sim$ 
$\pi^{\prime}[0, j]$ if $\pi[i] \in V_{1}, j=i+1$ and $\pi[0, i] \approx \pi^{\prime}[0, i]$, and define the formula:

$$
\text { SameAct' }:=\mathbf{G} \bigvee_{a \in \text { Act }} R p_{a} \text {. }
$$

Then a strategy $\sigma$ for Player 1 is observation-based if, and only if, it is $(\sim$ , SameAct')-strictly-uniform.

In this version, the relation is not reflexive, in particular plays ending in $V_{2}$ are linked to no play, making $R \varphi$ trivially true for any $\varphi$. This is the reason why we no longer need to mark positions of $V_{1}$ with the proposition $p_{1}$ and test whether we are in $V_{1}$ before we ask for some $p_{a}$ to hold in every reachable position.

Finally, notice that to embed the case of imperfect-recall for example, one would just have to replace $\approx$ with the appropriate relation.

\subsection{Games with epistemic condition}

Uniform strategies enable to express winning conditions that have epistemic features, the relevance of which is exemplified by the games with opacity condition studied in Maubert et al. [2011], . In that case, $R$ can represent a players' knowledge, or distributed knowledge between a group of players, or common knowledge, depending on the winning condition one wants to define.

Games with opacity condition are based on two-player imperfect-information arenas with a particular winning condition, called the opacity condition, which relies on the knowledge of the player with imperfect information. In such games, some positions are "secret" as they reveal a critical information that the imperfectinformation player wants to know (in the epistemic sense).

More formally, assume that a proposition $p_{S} \in A P$ represents the secret. Let $\mathcal{G}_{\text {inf }}=(\mathcal{G}, \sim)$ be an imperfect-information arena as described in Section 3.1, with a distinguished set of positions $S \subseteq V_{1}$ that denotes the secret. Let $\mathcal{G}=\left(V, E, v_{I}, \ell\right)$ be the arena with $\ell^{-1}\left(\left\{p_{S}\right\}\right)=S$ (positions labeled by $p_{S}$ are exactly positions $v \in S)$. The opacity winning condition is as follows.

The knowledge or information set of Player 1 after a finite play is the set of positions that she considers possible according to the observation she has: let $\rho \in$ Plays $_{*}$ be a finite play with last $(\rho) \in V_{1}$. The knowledge or information set of Player 1 after $\rho$ is $I(\rho):=\left\{\operatorname{last}\left(\rho^{\prime}\right) \mid \rho^{\prime} \in\right.$ Plays $\left._{*}, \rho \approx \rho^{\prime}\right\}$.

An infinite play is winning for Player 1 if there exists a finite prefix $\rho$ of this play whose information set is contained in $S$, i.e. $I(\rho) \subseteq S$, otherwise Player 2 wins. Again, strategies for Player 1 are required to be observation-based. It can easily be shown that:

\section{Theorem 2.}

A strategy $\sigma$ for Player 1 is winning if, and only if, $\sigma$ is $\left(\approx, \mathbf{F} R p_{S}\right)$-strictly-uniform. $A$ strategy $\sigma$ for Player 2 is winning if, and only if, $\sigma$ is $\left(\approx, \mathbf{G} \neg R p_{S}\right)$-fully-uniform.

For the second statement of Theorem 2, we make use of the notion of full uniformity because we are interested in the knowledge of Player 1 . We consider that 
she does not know what strategy Player 2 is playing, hence she may consider possible some plays that are observationally equivalent to her but not induced by this strategy.

On the other hand, for the first statement, we use strict uniformity but could have used full uniformity instead. Indeed, since the actions chosen by Player 1 are part of what she observes, she cannot consider possible a finite play that has not followed her strategy until the point $i$ considered. In fact, if $\pi$ is induced by $\sigma$ and $\pi[0, i] \approx \pi^{\prime}[0, i]$, then $\pi^{\prime}[0, i]$ is also induced by $\sigma$. The future of the play may not follow sigma, but this is not a problem here. Indeed, the property that we consider on equivalent plays, $p_{S}$, does not depend on the future.

Notice that though we chose to illustrate with an example, any winning condition that could be expressed as a formula of the linear temporal logic with one knowledge operator would fit in our setting.

\subsection{Non-interference}

Non-interference, as introduced by Goguen and Meseguer [1982], is a property evaluated on labelled transition systems which handle Boolean variables. Such systems are tuples $\left(S, \mathcal{I}, \mathcal{O}, \delta, s_{I}\right.$, Output $)$ where $S$ is the set of states, $\mathcal{I}=H \uplus L$ is a set of Boolean input variables partitioned into high security variables $H$ and low security variables $L, \mathcal{O}$ is the set of Boolean output variables, $\delta: S \times 2^{\mathcal{I}} \rightarrow S$ is the transition function that maps each pair of state and input variables valuation ${ }^{\mathrm{a}}$ to a next state, $s_{I}$ is the initial state, and Output $: S \rightarrow 2^{\mathcal{O}}$ is the output function that represents a mapping of states onto valuations of the Boolean output variables. We extend the transition function $\delta$ to $S \times\left(2^{\mathcal{I}}\right)^{*} \rightarrow S$ as expected: $\delta(s, \epsilon)=s$ and $\delta(s, u a)=\delta(\delta(s, u), a)$.

We define the $L$-equivalence, $\sim_{L}$ over $\left(2^{\mathcal{I}}\right)^{*}$ by $u \sim_{L} u^{\prime}$ whenever $u$ and $u^{\prime}$ have the same length and they coincide on the values of the low security input variables, i.e. for all $1 \leq i \leq|u|$, for all $l \in L, l \in u(i) \Leftrightarrow l \in u^{\prime}(i)$. Given an infinite sequence of inputs $w \in\left(2^{\mathcal{I}}\right)^{\omega}$, we abuse notation by writing $\operatorname{Output}(w)$ for the infinite sequence of output variables valuations encountered in the states along the execution of the system on input $w$. A system $\left(S, \mathcal{I}, \mathcal{O}, \delta, s_{I}\right.$, Output $)$ verifies the non-interference property if for any two finite sequences of inputs $w, w^{\prime} \in\left(2^{\mathcal{I}}\right)^{*}, w \approx_{L} w^{\prime}$ implies $\operatorname{Output}(w)=\operatorname{Output}\left(w^{\prime}\right)$. In other words, the valuations of high security variables have no consequence on the observation of the system.

A first natural problem is to decide the non-interference property of a system. A second more general problem is a control problem: we want to decide whether there is a way of restricting the set of input valuations along the executions, or equivalently to control the environment, so that the system is non-interfering. By constraining the applied restriction to be trivial, the former problem is a particular case of the latter. We can encode the control problem in our setting.

${ }^{a}$ we classically confuse valuations over a set $\mathrm{B}$ of Boolean variables with elements of $2^{B}$. 
Let $S y s=\left(S, \mathcal{I}, \mathcal{O}, \delta, s_{I}\right.$, Output $)$ be an instance of the problem, and write $\Sigma$ for $2^{\mathcal{I}}$ with typical elements $a, b, \ldots$ Without loss of generality, we can assume that $S y s$ is complete: every input valuation yields a transition. We define a two-player game arena that simulates the system, in which Player 1 fixes the environment, i.e. a subset of the possible inputs in the current state, and Player 2 chooses a particular input among those. More formally, let $\mathcal{G}_{S y s}=\left(V, E, v_{I}, \ell\right)$, with $V=V_{1} \uplus V_{2}$, $V_{1}=(\Sigma \uplus\{\epsilon\}) \times S$ and $V_{2}=S \times 2^{\Sigma}$. A position $(a, s) \in V_{1}$ denotes a situation where the system reaches state $s$ by an $a$-transition, and $(s, A) \in V_{2}$ denotes a situation where in state $s$, the set of possible inputs is $A$. The set of edges $E$ of the arena is the smallest set such that $(a, s) E(s, A)$ for all $s \in S, a \in \Sigma$ and $A \subseteq \Sigma$, and $(s, A) E(a, \delta(s, a))$ whenever $s \in S$ and $a \in A$. The initial position of the arena is $v_{I}=\left(\epsilon, s_{I}\right)$, and by assuming that $\left\{p_{o} \mid o \in 2^{\mathcal{O}}\right\} \subseteq A P$, we set $\ell(a, s)=\ell(s, A)=\left\{p_{\text {Output }(s)}\right\}$.

By writing $\iota$ for the canonical projection from $V_{1} \cup V_{2}$ onto $2^{\mathcal{I}}$ (that is $\iota\left(\epsilon, s_{I}\right)=$ $\iota(s, A)=\epsilon$ and $\iota(a, s)=a)$ and by extending $\iota$ to finite plays as expected, we let $\rho \equiv_{L} \rho^{\prime}$ hold whenever $\iota(\rho) \sim_{L} \iota\left(\rho^{\prime}\right)$. We now define the formula

$$
\text { SameOutput }:=\mathbf{G} \bigwedge_{p_{o} \in A P}\left(p_{o} \rightarrow R p_{o}\right)
$$

which captures the property that the valuations of output variables along $\equiv_{L^{-}}$ equivalent executions of the system coincide, and we can establish the following.

Theorem 3. There is a one-to-one correspondence between $\left(\equiv_{L}\right.$, SameOutput)strictly-uniform strategies of Player 1 and the controllers which ensure the noninterference property of the system.

In particular, the trivial strategy of Player 1 , where from any position $(a, s)$ she chooses to move to $(s, \Sigma)$, is ( $\equiv_{L}$, SameOutput)-strictly-uniform if, and only if, the system has the non-interference property.

Here we have to use the strict uniformity as we only want to consider the executions of the machine allowed by the control represented by the strategy.

Notice that in order to make this control problem more realistic, one would seek a maximal permissive strategy/controller so that environments as "large" as possible are computed, but this is out of the scope of the paper.

\subsection{Diagnosis and Prognosis}

Diagnosis has been intensively studied, in particular by the discrete-event systems community (see for example Sampath et al. [1995]; Cassandras and Lafortune [1999]; Yoo and Lafortune [2002], ). Informally, in this setting, a discrete-event system is diagnosable if any occurrence of a faulty event during an execution is eventually detected. More formally, diagnosability is a property of discrete-event systems which are structures of the form $S y s=\left(S, \Sigma, \Sigma_{o}, \Delta, s_{I}, F\right)$, with $S$ the set of states, $\Sigma$ the set of events, $\Sigma_{0} \subseteq \Sigma$ the observable events, $\Delta \subseteq S \times \Sigma \times S$ the transition relation, 
$s_{I}$ the initial state and $F \subseteq S$ the faulty states; we assume that once a faulty state is reached, only faulty states can be reached (the fault is persistent). We can rephrase this problem in our setting, with a single player simulating the system. Notice that since there is only one player, a strategy defines a unique infinite play.

Here we assume that $p_{f} \in A P$ represents the fact of being faulty. Let $\mathcal{G}_{\text {Sys }}=$ $\left(V, E, v_{I}, \ell\right)$, with $V_{1}=\emptyset, V_{2}=(\Sigma \uplus\{\epsilon\}) \times S,(a, s) E\left(b, s^{\prime}\right)$ whenever $\left(s, b, s^{\prime}\right) \in \Delta$, $v_{I}=\left(\epsilon, s_{I}\right)$, and $\ell(a, s)=\{f\}$ if $s \in F, \emptyset$ otherwise. We write $\rho \equiv_{\Sigma_{o}} \rho^{\prime}$ whenever the sequences of observable events underlying $\rho$ and $\rho^{\prime}$ are the same (these sequences are obtained from the sequences of positions in the play: for each position of the form $(a, s)$, keep its letter $a$ if $a \in \Sigma_{o}$, and delete it otherwise). In this game Player 1 never plays, all she does is look at Player 2 simulate the system. There is only one strategy for her, which is to do nothing, and all possible plays, representing all possible executions of Sys, are in the outcome of this strategy.

Theorem 4. Sys is diagnosable if, and only if, Player 1 has a $\left(\equiv_{\Sigma_{o}}, \mathbf{F} p_{f} \rightarrow \mathbf{F} R p_{f}\right)-$ fully-uniform strategy in $\mathcal{G}_{\text {Sys }}$.

Here again, since the outcome of the only possible strategy for Player 1 is the whole set of plays, we could equivalently choose full or strict uniformity.

Prognosis is a companion of diagnosis, but focuses on the ability to predict that a fault will occur. Prognosability-like properties can be defined in our setting. As an example, we aim at saying that a system is prognosable whenever the fact that a fault occurs in a system is known at least one step in advance. We define the following formula, which means that either a fault never occurs, or it occurs but we know it one step before it does.

$$
\text { Prognose } \left.:=\left(\neg p_{f}\right) \mathcal{W}\left(\neg p_{f} \wedge R \odot p_{f}\right)\right)
$$

Using the same framework as for diagnosis, we can propose:

Definition 7. A system Sys is prognosable if there is a ( $\equiv_{\Sigma_{o}}$, Prognose)-fullyuniform strategy for Player 1 in $\mathcal{G}_{\text {Sys }}$.

\subsection{Dependence Logic}

Dependence Logic is a flourishing topic introduced recently in Väänänen [2007]. It extends first order logic by adding atomic dependence formulas $\operatorname{dep}\left(t_{1}, \ldots, t_{n}\right)$, which express functional dependence of the term $t_{n}$ on the terms $t_{1}, \ldots, t_{n-1}$. A dependence atom $\operatorname{dep}\left(x_{0}, x_{1}\right)$ can be interpreted as "the value of $x_{1}$ depends only on the value of $x_{0}$ ", or "the value of $x_{1}$ is fully determined by the value of $x_{0}$ ". Evaluating a dependence between terms on a single assignment of the free variables is meaningless: in order to tell whether $t$ depends on $t^{\prime}$, one must vary the values of $t^{\prime}$ and see how the values of $t$ are affected. This is why a formula of Dependence Logic is evaluated on a first-order model $\mathcal{M}$ and a set of assignments for the free variables, called a team. If $t$ is a term, $\mathcal{M}$ a model and $s$ an assignment for the free 
variables in $t$, we note $\llbracket t \rrbracket_{s}^{\mathcal{M}} \in M$ the interpretation of $t$ in the model $\mathcal{M}$ with the assignment $s$.

Dependence Logic is inspired by Independence Friendly logic (IF logic), a logic defined by Hintikka and Sandu [1989]. Van Benthem gives in Van Benthem [2005] an imperfect-information evaluation game for IF logic, using a notion of uniform strategy that corresponds with the classic notion of imperfect-information or observationbased strategy.

For Dependence Logic, an evaluation game is also given in Väänänen [2007]. It is presented as a game with imperfect information, because strategies must verify some "uniformity constraint", which makes the game undetermined. However, the notion of uniform strategy in these games is not defined as "playing uniformly in positions of an information set", but rather as "playing such that, when positions of an information set are reached, some property uniformly holds in these positions". For this reason it is a notion of uniform strategy different from the one used in games with imperfect information. However, it is also based on a constraint on sets of related plays, and it is also an instance of our general notion of uniform strategies.

We present the evaluation game, the uniformity requirement and then show that this notion fits in our setting.

Let $\Phi$ be a sentence (formula with no free variable) of Dependence Logic in negation normal form, i.e. only atomic formulas can be negated, and let $\mathcal{M}$ be a first order model. $\mathcal{G}^{\mathcal{M}}(\varphi)$ is a two player game between Player 1 and Player 2; positions are of the form $(\varphi, n, s)$, where $\varphi$ is a subformula of $\Phi, n$ is the position in $\Phi$ of the first symbol of $\varphi$ and $s$ is an assignment whose domain contains the free variables of $\varphi$. The index $n$ is used to decide, given two positions containing the same dependence atom, whether they are the same syntactic subformulas of $\varphi$ or not. For a subformula $\varphi$, $\operatorname{len}(\varphi)$ is the number of symbols in $\varphi$. The game starts in position $(\varphi, 1, \emptyset)$ and the rules are as follows:

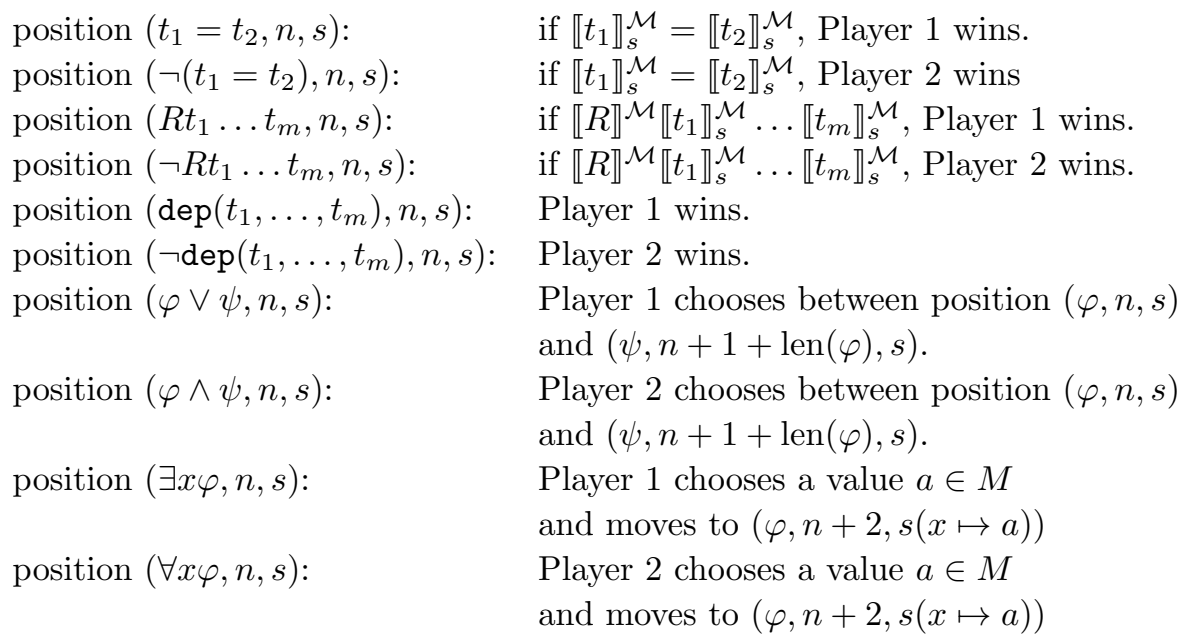




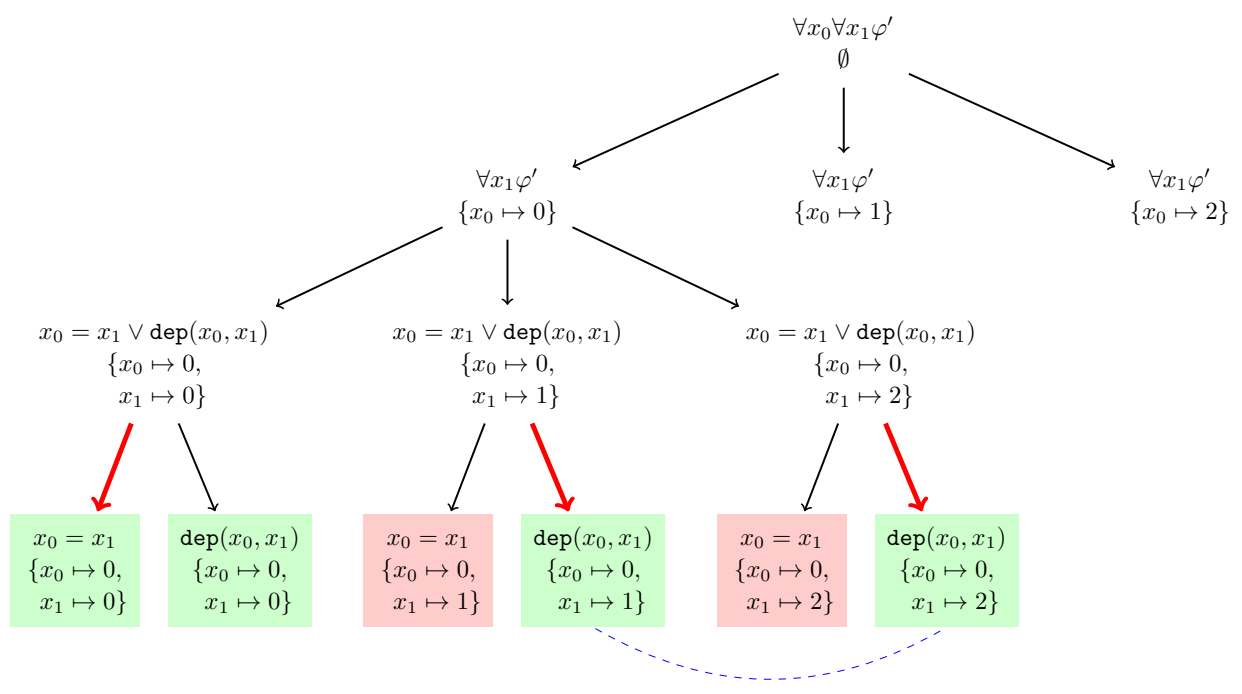

Fig. 1: Evaluation game for $\forall x_{0} \forall x_{1}\left(x_{0}=x_{1} \vee \operatorname{dep}\left(x_{0}, x_{1}\right)\right)$ with $M=\{0,1,2\}$

Figure 1 represents (a part of) the game for the evaluation of the Dependence Logic formula $\forall x_{0} \forall x_{1}\left(x_{0}=x_{1} \vee \operatorname{dep}\left(x_{0}, x_{1}\right)\right)$ on a model $\mathcal{M}$ with domain $M=$ $\{0,1,2\}$. This formula is not true in this model. Indeed, intuitively, if there are at least three elements in the domain, it is not because $x_{0}$ and $x_{1}$ do not have the same value that the value of $x_{0}$ determines the value of $x_{1}$ : there remain two possibilities for the value of $x_{1}$ that are not the one of $x_{0}$. So there should not be a winning strategy for Player 1 for the evaluation game to be correct.

In the first two rounds, Player 2 chooses a value for each of the universally quantified variables $x_{0}$ and $x_{1}$. Then Player 1 chooses a disjunct and we reach atomic formulas. Green positions are winning for Player 1, red ones are winning for Player 2. The red arrows indicate a strategy for Player 1 (we focus on the subtree for $x_{0}=0$, we assume that Player 1 plays the same way in the others). We see that this strategy is winning for Player 1, while the formula is not true in the model.

The problem comes from the fact that, as said earlier, a dependence atom must not be evaluated on a single assignment but on a set of assignments, a team. In the evaluation game, the team in which a dependence atom $\operatorname{dep}\left(t_{1}, \ldots, t_{n}\right)$ should be evaluated is the set of assignments in leafs that contain $\operatorname{dep}\left(t_{1}, \ldots, t_{n}\right)$ and are reached by the strategy. In the example, the assignments in the two leafs linked with the dashed line, $\left\{x_{0} \mapsto 0, x_{1} \mapsto 1\right\}$ and $\left\{x_{0} \mapsto 0, x_{1} \mapsto 2\right\}$, are thus part of the team in which $\operatorname{dep}\left(x_{0}, x_{1}\right)$ should be evaluated (there are more in the two other subtrees not shown here). Then we see that this strategy should not be allowed as while both leaves agree on the value of $x_{0}$, they do not agree on the value of $x_{1}$. This observation leads to defining a certain notion of uniform strategy. 
A strategy $\sigma$ for Player 1 is uniform in the sense of Väänänen [2007] if, for every two finite plays $\rho, \rho^{\prime} \in \operatorname{Out}(\sigma)$ such that $\operatorname{last}(\rho)=\left(\operatorname{dep}\left(t_{1}, \ldots, t_{m}\right), n, s, 1\right)$ and last $\left(\rho^{\prime}\right)=\left(\operatorname{dep}\left(t_{1}, \ldots, t_{m}\right), n, s^{\prime}, 1\right)$ contain the same (syntactically speaking) atomic dependence subformula, if $s$ and $s^{\prime}$ agree on $t_{1}, \ldots, t_{m-1}$, then they also agree on $t_{m}$. Then we have the expected property that a sentence $\varphi$ of Dependence Logic is true in a model $\mathcal{M}$ if Player 1 has a winning uniform strategy in $\mathcal{G}^{\mathcal{M}}(\varphi)$.

We characterize uniform strategies in the sense of Väänänen [2007] as uniform strategies in our sense. The game described above easily fits in our setting (we add loops on terminal positions so as to obtain infinite plays). Let $\Phi$ be a sentence of Dependence Logic, and $\mathcal{M}$ be a finite model. We call $G_{\Phi}^{\mathcal{M}}=\left(V, E, v_{I}\right)$ the evaluation game defined above. For each object $a \in M$ of the domain we use one atomic proposition $p_{a}$, and we also use the proposition $p_{d}$ to mark positions that contain dependence atoms. So we assume that $\left\{p_{a} \mid a \in M\right\} \uplus\left\{p_{d}\right\} \subset A P$, and we define $\mathcal{G}_{\Phi}^{\mathcal{M}}=\left(V, E, v_{I}, \ell\right)$, where the valuation $\ell$ is as follows :

$$
\begin{aligned}
\ell\left(\operatorname{dep}\left(t_{1}, \ldots, t_{m}\right), n, s\right) & =\left\{p_{a}, p_{d}\right\} \text { with } a=\llbracket t_{m} \rrbracket_{s}^{\mathcal{M}} \\
\ell\left({ }_{-}, n, s\right) & =\emptyset
\end{aligned}
$$

We define the equivalence relation $\simeq$ on finite plays as the smallest reflexive relation such that if there is $\varphi=\operatorname{dep}\left(t_{1}, \ldots, t_{m}\right)$ and $n$ s.t last $(\rho)=(\varphi, n, s)$, $\operatorname{last}\left(\rho^{\prime}\right)=\left(\varphi, n, s^{\prime}\right)$, and $\llbracket t_{i} \rrbracket_{s}^{\mathcal{M}}=\llbracket t_{i} \rrbracket_{s^{\prime}}^{\mathcal{M}}$ for $i=1, \ldots, n-1$, then $\rho \simeq \rho^{\prime}$. Now we define the formula

$$
\text { AgreeOnLast }:=\mathbf{G}\left(p_{d} \rightarrow \bigvee_{a \in M} R p_{a}\right)
$$

which expresses that whenever the current position contains a dependence atom $\operatorname{dep}\left(t_{1}, \ldots, t_{m}\right)$, it agrees with all equivalent finite plays on some value $a$ for $t_{m}$. Since equivalent plays are those ending in a position that has the same dependence atom and agrees on the first $m-1$ terms, it is easy to prove:

Theorem 5. A strategy $\sigma$ for Player 1 in $G_{\Phi}^{\mathcal{M}}$ is uniform if, and only if, it is $\left(\simeq\right.$, AgreeOnLast)-strictly-uniform in $\mathcal{G}_{\Phi}^{\mathcal{M}}$.

In this example again, the strict uniformity is needed, as we only want to catch leaves that are hit by the strategy. Also, note that here $\simeq$ is an equivalence because we take the reflexive closure of some transitive and reflexive relation. But as for observation based strategies, if we did not take the reflexive closure, we could avoid using the proposition $p_{d}$ and use the simpler formula

$$
\text { AgreeOnLast' }:=\mathbf{G} \bigvee_{a \in M} R p_{a}
$$

Indeed the relation would not be reflexive: in particular plays not ending in a dependence atom would not be linked to any play, and $R \varphi$ would trivially hold for any $\varphi$ in these plays; this is why testing whether we are in a dependence atom before enforcing that some $p_{a}$ must hold everywhere would no longer be needed. 


\subsection{Dependence logic and games with imperfect information}

As we said, the evaluation game for Dependence Logic presented in the previous subsection is said to be a game with imperfect information. We do not agree, because the difference between games with perfect information and games with imperfect information (at least in the perfect recall setting, it is not as clear otherwise, see Piccione and Rubinstein [1997]) lies in the fact that in the latter, some finite plays are related, in the sense that they are indistinguishable to one of the players, and this player must behave the same way in these related situations. Concerning the evaluation game for Dependence Logic, the difference with perfect-information games is that some plays are related, those ending in positions bound to the same atomic dependence formula $\operatorname{dep}\left(t_{1}, \ldots, t_{n}\right)$ with valuations agreeing on $t_{1}, \ldots, t_{n-1}$, and the valuations in these related positions must agree on $t_{n}$. So it is not that players should behave the same way in related situations, but rather that the players should have behaved in such a way that the valuations for $t_{n}$ are the same in related situations.

But it is true that there is a similarity between these two constraints on allowed strategies, as shown by looking at the formulas of the uniformity properties capturing observation-based strategies (SameAct) and uniform strategies in the sense of Dependence Logic (AgreeOnLast):

$$
\text { SameAct }=\mathbf{G}\left(p_{1} \rightarrow \bigvee_{a \in A c t} R \bigcirc p_{a}\right) \quad \text { and } \quad \text { AgreeOnLast }=\mathbf{G}\left(p_{d} \rightarrow \bigvee_{a \in M} R p_{a}\right)
$$

In the first case, the same thing must happen in equivalent situations, whereas in the second case, the same thing must hold in equivalent situations.

Neither semantics games for Dependence Logic are games with imperfect information in the classical sense, nor games with imperfect information can be easily described using the uniform strategy notion of Väänänen [2007], but both can be characterized in a very similar way with our notion of uniform strategies.

\section{Regular relations}

The binary relations used in the previous examples from the literature all fall into a particular class of relations, so-called rational ${ }^{\mathrm{b}}$ relations. They are characterized by some finite state machines called transducers Berstel [1979]. One way to see a transducer is to picture a nondeterministic automaton with two tapes, an input tape and an output tape. The transducer reads an input finite word on its input tape and writes out a finite word on its output tape. Notice that this machine is in general nondeterministic so that it may have several outputs for a given input word. Hence, transducers define binary relations.

Definition 8. A Finite State Transducer (FST) is a tuple $T=\left(Q, \Sigma, \Gamma, q_{i}, Q_{F}, \Delta\right)$, where $Q$ is a finite set of states, $\Sigma$ is the input alphabet, $\Gamma$ is the output alphabet,

\footnotetext{
${ }^{b}$ Note that rational relations do not relate at all with the game theoretic notion of rational players.
} 
$q_{i} \in Q$ is a distinguished initial state, $Q_{F} \subseteq Q$ is a set of accepting states, and $\Delta \subset Q \times(\Sigma \cup\{\epsilon\}) \times(\Gamma \cup\{\epsilon\}) \times Q$ is a finite set of transitions.

Intuitively, $\left(q, a, b, q^{\prime}\right) \in \Delta$ means that the transducer can move from state $q$ to state $q^{\prime}$ by reading $a$ and writing $b$ (both possibly $\epsilon$ ). We also define the extended transition relation $\Delta^{*}$, which is the smallest relation such that:

- for all $q \in Q,(q, \epsilon, \epsilon, q) \in \Delta^{*}$, and

- if $\left(q, w, w^{\prime}, q^{\prime}\right) \in \Delta^{*}$ and $\left(q^{\prime}, a, b, q^{\prime \prime}\right) \in \Delta$, then $\left(q, w \cdot a, w^{\prime} \cdot b, q^{\prime \prime}\right) \in \Delta^{*}$.

In the following, for $q, q^{\prime} \in Q, w \in \Sigma^{*}$ and $w^{\prime} \in \Gamma^{*}$, notation $q-\left[w / w^{\prime}\right] \rightarrow q^{\prime}$ means that $\left(q, w, w^{\prime}, q^{\prime}\right) \in \Delta^{*}$.

Definition 9. Let $T=\left(Q, \Sigma, \Gamma, q_{i}, Q_{F}, \Delta\right)$ be an $F S T$. The relation recognized by $T$ is

$$
[T]:=\left\{\left(w, w^{\prime}\right) \mid w \in \Sigma^{*}, w^{\prime} \in \Gamma^{*}, \exists q \in Q_{F}, q_{i}-\left[w / w^{\prime}\right] \rightarrow q\right\} .
$$

In other words, a pair $\left(w, w^{\prime}\right)$ is in the relation recognized by $T$ if there is an accepting execution of $T$ that reads $w$ and writes $w^{\prime}$.

Definition 10. Let $\Sigma$ and $\Gamma$ be two alphabets. A binary relation $\sim \subseteq \Sigma^{*} \times \Gamma^{*}$ is rational if it is recognized by an FST.

We now recall some basic properties of rational relations that will be useful later on. The first proposition regards intersection (rational relations are not closed under intersection in general) and the second one regards composition. The interested reader is referred to Berstel [1979] for technical details.

Proposition 1. Let $\Sigma$ and $\Gamma$ be two alphabets. Let $\leadsto \subseteq \Sigma^{*} \times \Gamma^{*}$ be a rational relation, and let $L$ and $L^{\prime}$ be two regular languages over $\Sigma$ and $\Gamma$ respectively. Then $\sim \cap\left(L \times L^{\prime}\right)$ is also a rational relation.

Let $\Sigma, \Sigma^{\prime}, \Sigma^{\prime \prime}$ be three alphabets. Let $\sim_{1} \subseteq \Sigma^{*} \times \Sigma^{* *}$ and $\sim_{2} \subseteq \Sigma^{* *} \times \Sigma^{\prime * *}$ be two binary relations.

Definition 11. The composition of $\sim_{1}$ and $\sim_{2}$ is $\sim_{1} \circ \sim_{2} \subseteq \Sigma^{*} \times \Sigma^{\prime \prime *}$ :

$$
\sim_{1} \circ \sim_{2}=\left\{\left(\rho, \rho^{\prime \prime}\right) \mid \exists \rho^{\prime} \in \Sigma^{\prime *}, \rho \sim_{1} \rho^{\prime} \text { and } \rho^{\prime} \sim_{2} \rho^{\prime \prime}\right\}
$$

Proposition 2. Elgot and Mezei [1965] If $\sim_{1}$ and $\sim_{2}$ are two rational relations recognized respectively by $T_{1}$ and $T_{2}$, then $\sim_{1} \circ \sim_{2}$ is also rational, and one can effectively build the transducer $T_{1} \circ T_{2}$, that recognizes $\sim_{1} \circ \sim_{2}$.

We close this section by taking two examples of binary relations involved in Section 3 and showing that they are rational. In fact, one can check that they all are.

Example 1. We first consider an example induced by the imperfect-information setting of Section 3.1. Let $\mathcal{G}_{i m p}=\left(V, E, v_{I}, \ell, \sim\right)$ be an imperfect-information game 


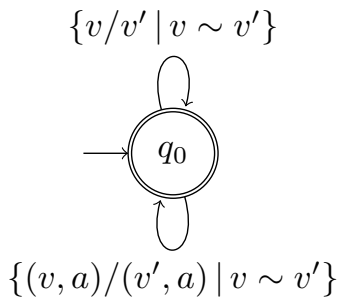

Fig. 2: $T_{o b s}$, an FST for the equivalence relation $\simeq$ of Sections 3.1 and 3.2.

arena as described in Section 3.1. Relation $\approx \subseteq$ Plays $_{*}^{2}$ is an observational equivalence over plays, generated by the equivalence $\sim$ between positions. Consider the FST $T_{o b s}$ depicted in Figure 2, with a unique initial state (ingoing arrow) that is also the final state (two concentric circles). It reads an input letter (a position) and outputs any position that is $\sim$-equivalent to it. This FST recognizes a relation $\simeq$ over $V^{*} \times V^{*}$, such that $\approx=\simeq \cap$ Plays $_{*}^{2}$. Because Plays $s_{*}$ is a regular language (one can see $\mathcal{G}$ as a finite state automaton), Proposition 1 gives that $\simeq \cap$ Plays $_{*}^{2}$ is also a rational relation, and in fact $\mathcal{G} \times T_{o b s} \times \mathcal{G}$ is a transducer that precisely recognizes $\approx$.

Example 2. Consider another binary relation that is also an equivalence, but induced by some alphabetic morphism $h: V \rightarrow \mathcal{O} \cup\{\epsilon\}$ : two plays $\rho$ and $\rho^{\prime}$ are equivalent, written $\rho \equiv_{\mathcal{O}} \rho^{\prime}$, whenever $h(\rho)=h\left(\rho^{\prime}\right)$. This example generalizes the one of Section 3.4 where the alphabetic morphism is a mere projection. In order to draw an FST for the relation $\equiv_{\mathcal{O}}$, we need to fix the set $\mathcal{O}$. Assume $\mathcal{O}$ has only two elements blue and pink so that any position in the game is either observed as $h(v)=$ blue or $h(v)=$ pink or unobserved (the case $h(v)=\epsilon$ ). The FST $T_{\mathcal{O}}$ that recognizes $\equiv_{\mathcal{O}}$ is drawn in Figure 3 . Once again, one should take the product of $T_{\mathcal{O}}$ with the game arena to restrict the relation to Plays $*$ $\times$ Plays $_{*}$. Remark that contrary to the case of equivalence $\approx$ in Figure 2 , the equivalence $\equiv_{\mathcal{O}}$ does not preserve the length of plays.

Example 1 is an example of a simple transducer, but in fact all relations of Section 3 can be recognized by the transducer given in Example 2, for an appropriate alphabetic morphism. In the next section, we will only consider rational relations over plays, i.e. the relation $\sim$ in the model is recognized by some FST.

\section{Automated synthesis of fully-uniform strategies}

In this section, we study the problem of synthesizing fully-uniform strategies. We restrict to finite arenas. Motivated by Section 4, we only consider rational relations, and as a consequence we always assume that the semantic relation between plays is 


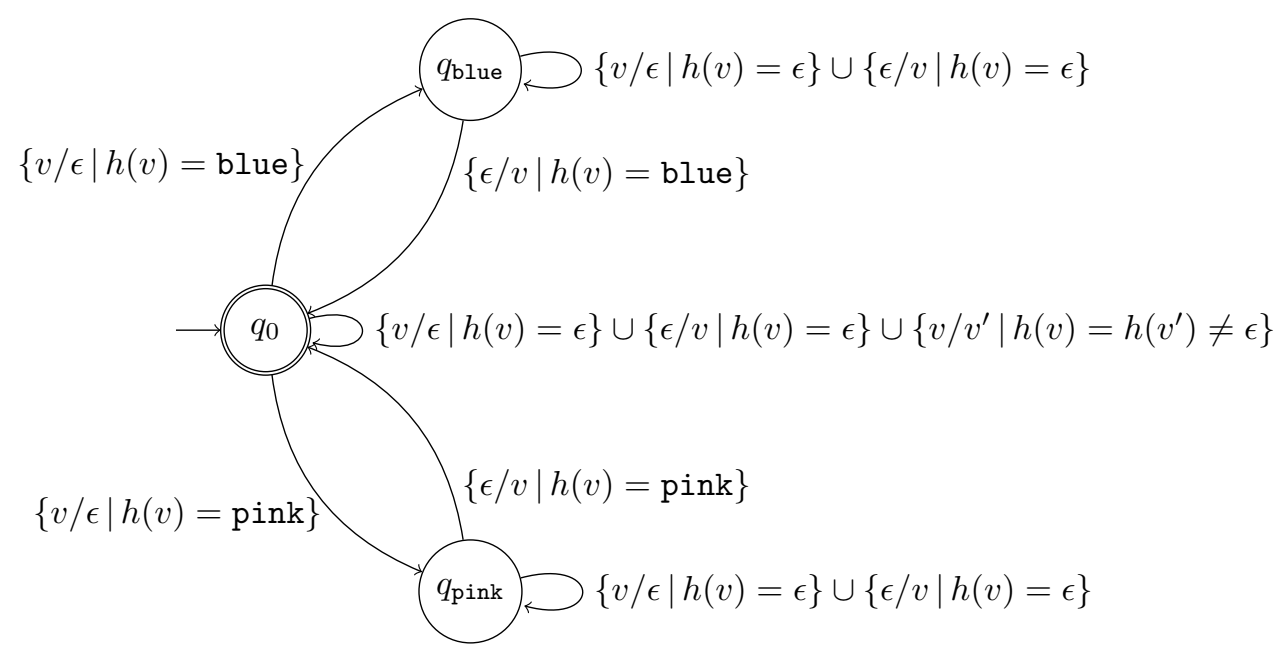

Fig. 3: $T_{\mathcal{O}}$, an FST for the equivalence relation $\equiv_{\mathcal{O}}$.

described by a finite state transducer $T$. Still, when it is clear from the context, we write $\sim$ instead of $[T]$. Also, because we only consider full uniformity and no longer the strict one, it is understood in the semantics of a formula that the universe $\Pi$ is the set of plays of the considered game, hence we omit it when it is clear from the context. Furthermore, we will sometimes make the semantic relation between plays explicit. All these conventions yield a notation of the form $\pi, i \models \sim \varphi$ meaning that Plays $_{\omega}, \pi, i=\varphi$ with the $R$-modality semantics based on the binary relation $\leadsto$.

Finally, in this section, the size $|\mathcal{G}|$ of an arena $\mathcal{G}$ is the number of positions, the size $|T|$ of a transducer $T$ is the number of states, and $|\varphi|$ is the size of formula $\varphi$.

Definition 12. For each $n \in \mathbb{N}$, we define the decision problem $\mathrm{FUS}_{n}$ by:

$\operatorname{FUS}_{n}:=\left\{(\mathcal{G}, T, \varphi) \mid \mathcal{G}\right.$ is an arena, $([T], \varphi)$ is a uniformity property with $\varphi \in \mathcal{L}_{n}$ and there is a $([T], \varphi)$-fully-uniform strategy for Player 1$\}$

The fully-uniform strategy problem is FUS: $=\bigcup_{n \in \mathbb{N}} \mathrm{FUS}_{n}$

For an instance $(\mathcal{G}, T, \varphi)$ of FUS, its size is defined as $|(\mathcal{G}, T, \varphi)|:=|\mathcal{G}|+|T|+|\varphi|$.

Theorem 6. $\mathrm{FUS}_{n}$ is in 2-ExPTIME for $n \leq 2$, and in $n$-ExPtime for $n>2$.

Corollary 1. The fully-uniform strategy problem is decidable.

The rest of this section is dedicated to the proof of Theorem 6 . We describe a powerset construction for a new arena (Section 5.1) and a way to lift the semantic relation between plays to this powerset construction (Section 5.2). Next we show how to exploit this construction to reduce membership in $\mathrm{FUS}_{n+1}$ to membership in $\mathrm{FUS}_{n}$ (Section 5.3). 


\subsection{Powerset arena}

In games with imperfect information, the information set of a player after a finite play is the set of positions that are consistent with what she has observed. We define a similar notion in our setting, and we show that the rationality assumption on the relation is sufficient to compute information sets and build a powerset construction arena in which formulas of the kind $R \varphi$ where $\varphi \in$ LTL can be evaluated positionally.

Definition 13. Let $\mathcal{G}$ be an arena, $\sim \subseteq$ Plays $_{*}^{2}$ and $\rho \in$ Plays $_{*}$. The information set after the finite play $\rho$ is the set of terminating positions of related finite plays. Formally, $I(\rho)=\left\{v^{\prime} \mid \exists \rho^{\prime} \cdot v^{\prime} \in\right.$ Plays $\left._{*}, \rho \sim \rho^{\prime} \cdot v^{\prime}\right\}$.

For an arena $\mathcal{G}$ and a transducer $T$ over Plays $_{*}$, we construct a powerset arena $\widehat{\mathcal{G}}$ in which formulas of the form $R \varphi$ can be evaluated positionally when $\varphi \in \mathcal{L}_{0}$.

Unlike classic powerset constructions Reif [1984]; Chatterjee et al. [2006], , in our setting, the new information set after a move in the game cannot be computed knowing only the previous information set and the new position. To compute information sets, we need to simulate the nondeterministic execution of $T$, taking as an input the sequence of positions played and writing as output the related plays. This is why in our construction, positions do not contain directly information sets, but rather we add in the positions of the game sufficient information on the current configuration of the transducer.

More precisely, two things are necessary: the set of states the transducer may be in after reading the sequence of positions played so far, plus for each of these states the set of possible last positions written on the output tape (because of nondeterminacy, different executions can end up in configurations with same state but different last letters on the output tape). We only need to remember the last letter on the output tape, and not the whole tape, because the information set which we aim at computing is just the set of the last positions of related plays.

So positions are of the form $(v, S, L a s t)$, where $S \subseteq Q$ is the set of possible current states of $T$, and Last $: S \rightarrow \mathcal{P}(V)$ associates to a state $q \in S$ the set of the possible last positions on the output tape of $T$ if the current state is $q$. The transitions in this arena follow the ones in $\mathcal{G}$, we just have to maintain the additional information about the configuration of the transducer. In order to define the initial position $\widehat{v}_{I}=\left(v_{I}, S_{I}\right.$, Last $\left._{I}\right)$ of $\widehat{\mathcal{G}}$ we need to simulate the execution of $T$ starting from its initial state and reading $v_{I}$. To do so, we introduce an artificial position $\widehat{v}_{-1}$ that initializes the transducer before reading the first position of a play. Concretely, $\widehat{v}_{-1}=\left(v_{-1}, S_{-1}\right.$, Last $\left._{-1}\right)$, with $v_{-1} \notin V$ a fresh position, $S_{-1}=\left\{q_{i}\right\}$ because before starting the transducer is in its initial state, and Last $_{-1}\left(q_{i}\right)=\emptyset$ because nothing is written on the output tape.

Definition 14. Let $\mathcal{G}=\left(V, E, v_{I}, \ell\right)$ be an arena and $T=\left(Q, V, q_{i}, Q_{F}, \Delta\right)$ be an FST such that $[T] \subseteq$ Plays $_{*}^{2}$. We define the arena $\widehat{\mathcal{G}}=\left(\widehat{V}, \widehat{E}, \widehat{v}_{I}, \widehat{\ell}\right)$ by: 
- $\widehat{V}=V \times \mathcal{P}(Q) \times(Q \rightarrow \mathcal{P}(V)) \uplus\left\{\widehat{v}_{-1}\right\}$

- $(u, S$, Last $) \widehat{\rightarrow}\left(v, S^{\prime}\right.$, Last $\left.^{\prime}\right)$ if

$-u=v_{-1}$ and $v=v_{I}$, or $u \rightarrow v$,

- $S^{\prime}=\left\{q^{\prime} \mid \exists q \in S, \exists \lambda^{\prime} \in V^{*}, q-\left[v / \lambda^{\prime}\right] \rightarrow q^{\prime}\right\}$ and

- $\operatorname{Last}^{\prime}\left(q^{\prime}\right)=\left\{v^{\prime} \mid \exists q \in S, \exists \lambda^{\prime} \in V^{*}, q-\left[v / \lambda^{\prime} \cdot v^{\prime}\right] \rightarrow q^{\prime}\right.$, or $q-[v / \epsilon] \rightarrow$ $q^{\prime}$ and $\left.v^{\prime} \in \operatorname{Last}(q)\right\}$

- $\widehat{v}_{I}$ is the only $\widehat{v} \in \widehat{V}$ such that $\widehat{v}_{-1} \widehat{\rightarrow} \widehat{v}$.

- $\widehat{\ell}(\widehat{v})=\ell(v)$ if $\widehat{v}=(v, S$, Last $)$.

Notice that $\widehat{\mathcal{G}}$ has size $|\widehat{\mathcal{G}}|=O\left(|\mathcal{G}| \times 2^{|T|} \times 2^{|T||\mathcal{G}|}\right)$.

Regarding the definition of transitions, the first point means that transitions in $\widehat{E}$ follow those in $E$, except for the only transition leaving $\widehat{v}_{-1}$, that is used to define $\widehat{v}_{I}$. The second point for the definition of $S^{\prime}$ expresses that when we move from $u$ to $v$ in $\mathcal{G}$, we give $v$ as an input to the transducer. So the set of states the transducer can be in after this move is exactly the set of states that can be reached from one of the previous possible states by reading $v$ and writing some sequence of positions (possibly $\epsilon$ ). We use the notation $\lambda$ because without loss of generality one could assume that the transducer can only output sequences of positions that form a valid path in the game graph. But it is not important here, the assumption that $[T] \subseteq$ Plays $_{*}^{2}$ is sufficient. Finally, the third point for the definition of Last $^{\prime}$ captures that if some position $v^{\prime}$ is at the end of the output tape after the transducer read $v$ and reached $q^{\prime}$, it is either because while reading $v$ the last letter it wrote is $v^{\prime}$, or it wrote nothing and $v^{\prime}$ was already the end of the output tape before reading $v$.

To finish with, $\widehat{v}_{I}$ is the only successor of $\widehat{v}_{-1}$, and the valuation of a position in the powerset construction is the valuation of the underlying position in the original arena.

Remark 1. We need to clarify the following definitions:

- $S^{\prime}=\left\{q^{\prime} \mid \exists q \in S, \exists \lambda^{\prime} \in V^{*}, q-\left[v / \lambda^{\prime}\right] \rightarrow q^{\prime}\right\}$ and

- $\operatorname{Last}^{\prime}\left(q^{\prime}\right)=\left\{v^{\prime} \mid \exists q \in S, \exists \lambda^{\prime} \in V^{*}, q-\left[v / \lambda^{\prime} \cdot v^{\prime}\right] \rightarrow q^{\prime}\right.$, or $q-[v / \epsilon] \rightarrow q^{\prime}$ and $v^{\prime} \in$ $\operatorname{Last}(q)\}$

Indeed, in each one, there can be infinitely many such $\lambda^{\prime}$ because of transitions that read nothing on the input tape. Still, $S^{\prime}$ and $L a s t^{\prime}$ can be computed in linear time in the size of $\Delta$. To do so, for each $q$ in $S$, we compute $S_{q, v}=\left\{\left(q^{\prime}, v^{\prime}\right) \mid \exists \lambda^{\prime} \in\right.$ $V^{*}, q-\left[v / \lambda^{\prime} \cdot v^{\prime}\right] \rightarrow q^{\prime}$, or $q-[v / \epsilon] \rightarrow q^{\prime}$ and $\left.v^{\prime} \in \operatorname{Last}(q)\right\} . S^{\prime}$ and Last $^{\prime}$ can be easily reconstructed from $\cup_{q \in S} S_{q, v}$. For $q \in S$, computing $S_{q, v}$ can be done by depth-first search, by first reading $v$ and then only $\epsilon$, and remembering the last output. The search can be stopped when we reach a state that has already been visited.

Let us take an arena $\mathcal{G}$ and an FST $T$ such that $[T] \subseteq$ Plays $_{*}^{2}$. For a position $\widehat{v}$ of $\widehat{\mathcal{G}}$, we will access the different components of the position with the notations $\widehat{v} . v, \widehat{v} . S, \widehat{v}$. Last. 
There is a natural mapping $f:$ Plays $_{\omega} \rightarrow \widehat{\operatorname{Plays}}_{\omega}$ : for an infinite play $\pi \in \mathcal{G}$, we define $f(\pi)$ as the only play $\widehat{\pi}$ in $\widehat{\operatorname{Plays}}_{\omega}$ such that $\widehat{\pi}[0] . v \cdot \widehat{\pi}[1] \cdot v \cdot \widehat{\pi}[2] . v \ldots=\pi$. This is well defined because from a position $\widehat{u}=(u, S$, Last $)$ in $\widehat{V}$, for $v \in V$ such that $u \rightarrow v$, there is a unique move $\widehat{u} \widehat{\rightarrow} \widehat{v}$ such that $\widehat{v} \cdot v=v$. It is easy to see that $f$ is a bijection. From now on we will slightly abuse notations: for a play $\pi \in$ Plays $_{\omega}$, $\widehat{\pi}$ will denote $f(\pi)$, and for $\widehat{\pi} \in \widehat{\text { Plays }}_{\omega}, \pi$ will denote $f^{-1}(\widehat{\pi})$. idem for finite plays.

Definition 15. Let $\widehat{v}=(v, S$, Last $) \in \widehat{V}$ be a position in the powerset construction. Its local information set $\widehat{v} . I$ is defined by:

$$
\widehat{v} . I:=\bigcup_{q \in S \cap Q_{F}} \operatorname{Last}(q)
$$

Definition 15 means that a position $v^{\prime}$ is in the information set after a play $\widehat{\rho}$ if and only if there is an execution of the transducer on the word seen so far that terminates in an accepting state with $v^{\prime}$ the last output. Actually, the local information sets correspond to the real information sets as expressed by the following proposition.

Proposition 3. For all $\widehat{\rho} \in \widehat{\operatorname{Plays}}_{*}$, last $(\widehat{\rho}) . I=I(\rho)$.

The rest of this subsection is dedicated to the proof of Proposition 3.

Lemma 1. Let $\widehat{\rho} \in \widehat{\operatorname{Plays}}_{*}$, and let $\widehat{v}:=\operatorname{last}(\widehat{\rho})$. Then, $\widehat{v} \cdot S=\left\{q \mid \exists \lambda^{\prime} \in V^{*}, q_{i}-\right.$ $\left.\left[\rho / \lambda^{\prime}\right] \rightarrow q\right\}$, and for each $q \in \widehat{v} . S$, $\widehat{v} . \operatorname{Last}(q)=\left\{v^{\prime} \mid \exists \lambda^{\prime} \in V^{*}, q_{i}-\left[\rho / \lambda^{\prime} \cdot v^{\prime}\right] \rightarrow q\right\}$.

\section{Proof.}

The proof is by induction on $\widehat{\rho}$.

Case $\widehat{\boldsymbol{v}}_{\boldsymbol{I}}$. We note $\widehat{v}_{I}=\left(v_{I}, S_{I}\right.$, Last $\left._{I}\right)$, and start with the left-right inclusions for both equalities. Let $q^{\prime} \in S_{I}$ and $v^{\prime} \in \operatorname{Last}_{I}(q)$. By definition of Last $_{I}$ and $S_{I}$ there is a $q$ in $S_{-1}=\left\{q_{i}\right\}$ (so $q=q_{i}$ ) and a $\lambda^{\prime} \in V^{*}$ such that $q_{i}-\left[v_{I} / \lambda^{\prime}\right] \rightarrow q^{\prime}$, which proves the first inclusion. Since Last $_{-1}\left(q_{i}\right)=\emptyset$ by definition, the case $\lambda^{\prime}=\epsilon$ is not possible. So there exists $\lambda^{\prime \prime}$ such that $\lambda^{\prime}=\lambda^{\prime \prime} \cdot v^{\prime}$, which gives us the second inclusion.

The proofs for the two right-left inclusions are straightforward applications of the definitions of $S_{I}$ and Last $_{I}$.

Case $\widehat{\boldsymbol{\rho}} \cdot \widehat{\boldsymbol{u}} \cdot \widehat{\boldsymbol{v}}, \widehat{\boldsymbol{\rho}} \in \widehat{\text { Plays }_{*}} \cup\{\boldsymbol{\epsilon}\}$. We note $\widehat{u}=(u, S$, Last $)$ and $\widehat{v}=\left(v, S^{\prime}\right.$, Last $\left.^{\prime}\right)$. For the two left-right inclusions, let $q^{\prime} \in S^{\prime}$ and $v^{\prime} \in \operatorname{Last}^{\prime}\left(q^{\prime}\right)$. By definition of $S^{\prime}$ and Last there is a $q$ in $S$ and a $\lambda_{1}^{\prime} \in V^{*}$ such that $q-\left[v / \lambda_{1}^{\prime}\right] \rightarrow q^{\prime}$, and either $\lambda_{1}^{\prime}=\lambda^{\prime} \cdot v^{\prime}$ for some $\lambda^{\prime}$, or $\lambda_{1}^{\prime}=\epsilon$ and $v^{\prime} \in \operatorname{Last}(q)$. By induction hypothesis, we have that $S=\left\{q \mid \exists \lambda^{\prime} \in V^{*}, q_{i}-\left[\rho \cdot u / \lambda^{\prime}\right] \rightarrow q\right\}$, so there exists $\lambda_{2}^{\prime} \in V^{*}$ such that $q_{i}-\left[\rho \cdot u / \lambda_{2}^{\prime}\right] \rightarrow q$, and by transitivity, $q_{i}-\left[\rho \cdot u \cdot v / \lambda_{2}^{\prime} \cdot \lambda_{1}^{\prime}\right] \rightarrow q^{\prime}$. This proves the first left-right inclusion. For the second one we split the two cases for $\lambda_{1}^{\prime}$. 
- If $\lambda_{1}^{\prime}=\lambda_{3}^{\prime} \cdot v^{\prime}$ for some $\lambda_{3}^{\prime}$, then by transitivity we have $q_{i}-\left[\rho \cdot u \cdot v / \lambda_{2}^{\prime} \cdot \lambda_{3}^{\prime} \cdot v^{\prime}\right] \rightarrow$ $q^{\prime}$, which proves the second left-right inclusion.

- If $\lambda_{1}^{\prime}=\epsilon$, then $v^{\prime} \in \operatorname{Last}(q)$. By induction hypothesis there is some $\lambda_{3}^{\prime}$ such that $q_{i}-\left[\rho \cdot u / \lambda_{3}^{\prime} \cdot v^{\prime}\right] \rightarrow q$. By transitivity we obtain $q_{i}-\left[\rho \cdot u \cdot v / \lambda_{3}^{\prime} \cdot v^{\prime}\right] \rightarrow q^{\prime}$, which also proves the second left-right inclusion.

Now for the first right-left inclusion, take $q^{\prime}$ and $\lambda^{\prime}$ such that $q_{i}-\left[\rho \cdot u \cdot v / \lambda^{\prime}\right] \rightarrow q^{\prime}$. Necessarily there exist $\lambda_{1}^{\prime}, \lambda_{2}^{\prime}$ and $q$ such that $q_{i}-\left[\rho \cdot u / \lambda_{1}^{\prime}\right] \rightarrow q, q-\left[v / \lambda_{2}^{\prime}\right] \rightarrow q^{\prime}$ and $\lambda_{1}^{\prime}$. $\lambda_{2}^{\prime}=\lambda^{\prime}$. By induction hypothesis $q \in S$, so by definition of $S^{\prime}, q^{\prime} \in S^{\prime}$. For the second right-left inclusion, take $q^{\prime} \in S^{\prime}$, and take $v^{\prime}$ and $\lambda^{\prime}$ such that $q_{i}-\left[\rho \cdot u \cdot v / \lambda^{\prime} \cdot v^{\prime}\right] \rightarrow q^{\prime}$. Again, necessarily there exist $\lambda_{1}^{\prime}, \lambda_{2}^{\prime}$ and $q$ such that $q_{i}-\left[\rho \cdot u / \lambda_{1}^{\prime}\right] \rightarrow q, q-\left[v / \lambda_{2}^{\prime}\right] \rightarrow q^{\prime}$ and $\lambda_{1}^{\prime} \cdot \lambda_{2}^{\prime}=\lambda^{\prime} \cdot v^{\prime}$. By induction hypothesis $q \in S$. We distinguish two cases.

- If $\lambda_{2}^{\prime}=\epsilon$, then $\lambda_{1}^{\prime}=\lambda^{\prime} \cdot v^{\prime}$, hence $q_{i}-\left[\rho \cdot u / \lambda^{\prime} \cdot v^{\prime}\right] \rightarrow q$. By induction hypothesis, $v^{\prime} \in \operatorname{Last}(q)$, so by definition of $\operatorname{Last}^{\prime}$, because $q \in S$ and $q-[v / \epsilon] \rightarrow q^{\prime}$, we obtain $v^{\prime} \in \operatorname{Last}^{\prime}\left(q^{\prime}\right)$.

- If $\lambda_{2}^{\prime}=\lambda_{3}^{\prime} \cdot v^{\prime}$ for some $\lambda_{3}^{\prime}$, then by definition of Last $^{\prime}$, because $q \in S$, we have $v^{\prime} \in \operatorname{Last}^{\prime}\left(q^{\prime}\right)$.

This finishes the induction.

We can now terminate the proof of Proposition 3: Let $\widehat{\rho} \in \widehat{\operatorname{Plays}}_{*}$, and let $\widehat{v}=\operatorname{last}(\widehat{\rho})$ be of the form $\widehat{v}=(v, S$, Last). We remind that (Definition 13) $I(\rho)=\left\{v^{\prime} \in V \mid \exists \rho^{\prime} \cdot v^{\prime} \in\right.$ Plays $\left._{*}, \rho \leadsto \rho^{\prime} \cdot v^{\prime}\right\}$.

We start with the left-right inclusion. Let $v^{\prime} \in \widehat{v} . I$. By definition, $\widehat{v} . I=$ $\bigcup_{q \in S \cap Q_{F}} \operatorname{Last}(q)$, so $v^{\prime} \in \operatorname{Last}(q)$ for some $q \in S \cap Q_{F}$. By Lemma 1, there exists $\lambda^{\prime} \in V^{*}$ such that $\left.q_{i} \dashv \rho / \lambda^{\prime} \cdot v^{\prime}\right] \rightarrow q$, and because $q \in Q_{F}$, we have that $\left(\rho, \lambda^{\prime} \cdot v^{\prime}\right) \in[T]$, which implies that $\rho \sim \lambda^{\prime} \cdot v^{\prime}$. Since $\sim \subseteq$ Plays $_{*}^{2}, \lambda^{\prime} \cdot v^{\prime} \in$ Plays $_{*}$, hence $v^{\prime} \in I(\rho)$.

For the right-left inclusion, take $v^{\prime} \in I(\rho)$. There exists $\rho^{\prime}$ such that $\rho^{\prime} \cdot v^{\prime} \in$ Plays $_{*}$ and $\rho \sim \rho^{\prime} \cdot v^{\prime}$. By definition of $T$, there exists $q \in Q_{F}$ such that $q_{i}-\left\{\rho / \rho^{\prime} \cdot v^{\prime}\right] \rightarrow$ $q$. By Lemma $1, q \in S$, and $v^{\prime} \in \operatorname{Last}(q)$. Since $q \in S \cap Q_{F}, v^{\prime} \in \widehat{v} . I$.

\subsection{Lifting transducers}

Let $\mathcal{G}$ be an arena, $T$ an FST such that $[T] \subseteq$ Plays $_{*}^{2}$, and let $\widehat{\mathcal{G}}=\operatorname{Power}(\mathcal{G}, T)$. We describe how to build a transducer $\widehat{T}$ that lifts $[T] \subseteq$ Plays $_{*} \times$ Plays $_{*}$ to $\widehat{\operatorname{Plays}}_{*} \times \widehat{\text { Plays }}_{*}$.

We note $T \downarrow$ for the deterministic transducer that computes $f$, the bijection that maps a play $\hat{\rho} \in \widehat{\text { Plays }}_{*}$ to the underlying play $\rho \in$ Plays $_{*}$, and $T \uparrow$ for the deterministic transducer that computes $f^{-1}$. Both are easily built from $\widehat{\mathcal{G}}$, and $|T \downarrow|=|T \uparrow|=O(|\widehat{\mathcal{G}}|)$.

Definition 16. The lift of transducer $T$ is $\widehat{T}=T \downarrow \circ T \circ T \uparrow$. 
Notice that $|\widehat{T}|=O(|\widehat{\mathcal{G}}| \times|T| \times|\widehat{\mathcal{G}}|)$.

In the following we let $\leadsto=[T]$ and $\widehat{\sim}=[\widehat{T}]$. The following proposition follows directly from the definitions of $\widehat{\mathcal{G}}$ and $\widehat{T}$ :

Proposition 4. For every $\varphi \in \mathcal{L}, \pi \in$ Plays $_{\omega}, i \geq 0$,

$$
\pi, i \models \sim \varphi \text { iff } \widehat{\pi}, i \models \approx \varphi
$$

\subsection{R-elimination}

We establish that given an instance of $\mathrm{FUS}_{n+1}$, we can build in exponential space and time an equivalent instance of $\mathrm{FUS}_{n}$.

\section{Proposition 5.}

For all instance $(\mathcal{G}, T, \varphi)$ of $\mathrm{FUS}_{n+1}$, there exists an instance $\left(\mathcal{G}^{\prime}, T^{\prime}, \varphi^{\prime}\right)$ of $\mathrm{FUS}_{n}$ computable in time exponential in $|(\mathcal{G}, T, \varphi)|$ such that:

- $(\mathcal{G}, T, \varphi) \in \mathrm{FUS}_{n+1}$ iff $\left(\mathcal{G}^{\prime}, T^{\prime}, \varphi^{\prime}\right) \in \mathrm{FUS}_{n}$

- $\left|\mathcal{G}^{\prime}\right|=O\left(2^{(|\mathcal{G}|+|T|)^{2}}\right)$

- $\left|T^{\prime}\right|=O\left(2^{O(|\mathcal{G}|+|T|)^{2}}\right)$

- $\left|\varphi^{\prime}\right|=O(|\varphi|)$

The rest of the section is dedicated to the proof of Proposition 5. Let $(\mathcal{G}, T, \varphi)$ be an instance of $\mathrm{FUS}_{n+1}$.

Lemma 2. Let $\pi \in$ Plays $_{\omega}, i \geq 0$, and $\varphi$ be an LTL-formula.

$$
\pi, i \models R \varphi \text { iff } \mathcal{G}, u \models \varphi \text { for all } u \in I(\pi[0, i]) .
$$

Proof. We start with the left-right implication. Suppose that $\pi, i=R \varphi$ holds, and take $u \in I(\pi[0, i])$. We need to prove that $\mathcal{G}, u \models \varphi$. To do so, we take $\pi^{\prime} \in$ $\operatorname{Traces}_{\omega}(u)$ an infinite trace starting in $u$ and we prove that $\pi^{\prime}, 0 \models \varphi$. Since $u \in I(\pi[0, i])$, by definition of the information set, there exists $\rho \cdot u \in$ Plays $_{*}$ such that $\pi[0, i] \sim \rho \cdot u$. We let $j=|\rho|$ and $\pi^{\prime \prime}=\rho \cdot \pi^{\prime}$ be such that $\pi^{\prime \prime}[j]=u$. Clearly, $\pi^{\prime \prime} \in$ Plays $_{\omega}$, and $\pi[0, i] \sim \pi[0, j]$. Since $\pi, i \models R \varphi$ holds, we have that $\pi^{\prime \prime}, j \models \varphi$. And because $\varphi \in \mathrm{LTL}$, the fact that it holds at some point of a trace only depends on the future of this point, hence $\pi^{\prime \prime}[j, \infty], 0 \models \varphi$, i.e. $\pi^{\prime}, 0 \models \varphi$.

For the right-left implication, suppose that $\mathcal{G}, u \models \varphi$ for all $u \in I(\pi[0, i])$, and take $\pi^{\prime} \in$ Plays $_{\omega}, j \geq 0$ such that $\pi[0, i] \sim \pi^{\prime}[0, j]$. We have that $\pi^{\prime}[j] \in I(\pi[0, i])$, so $\mathcal{G}, \pi^{\prime}[j] \models \varphi$. Because $\pi^{\prime}[j, \infty]$ is in $\operatorname{Traces}_{\omega}\left(\pi^{\prime}[j]\right)$, we have that $\pi^{\prime}[j, \infty], 0 \models \varphi$, hence $\pi^{\prime}, j \models \varphi$.

Lemma 3. Let $\widehat{\pi} \in \widehat{\operatorname{Plays}}_{\omega}, i \geq 0$, and let $\varphi$ be an LTL-formula.

$$
\widehat{\pi}, i \models \approx \operatorname{Riff} \mathcal{G}, u \models \varphi \text { for all } u \in \widehat{\pi}[i] . I .
$$


Proof. Let $\widehat{\pi} \in \widehat{\operatorname{Plays}}_{\omega}$ and $i \geq 0$. By Proposition 4, for any $\varphi \in$ LTL, $\widehat{\pi}, i \models \approx$ $R \varphi$ iff $\pi, i=_{\sim} R \varphi$, and by Lemma $2, \pi, i \models_{\sim} R \varphi$ iff $\mathcal{G}, u \models \varphi$ for all $u \in I(\pi[0, i])$. Now, by Proposition $3, \widehat{\pi}[i] . I=I(\pi[0, i])$, which concludes the proof.

We now define how formulas of the kind $R \varphi$ can be replaced by new atomic propositions, and how positions of the powerset arena can be marked with these new propositions: To an arena $\mathcal{G}$, a formula $\varphi \in \mathcal{L}$ and a subformula $R \psi \in \mathcal{L}_{1} \cap \operatorname{Sub}(\varphi)$ (if any), we associate a fresh atomic proposition $p_{R \psi}$ that occurs neither in $\mathcal{G}$ nor in $\varphi$.

Definition 17. For $\varphi \in \mathcal{L}_{n+1}$, we define $\widehat{\varphi}:=\varphi\left[p_{R \psi} / R \psi \mid R \psi \in \mathcal{L}_{1} \cap S u b(\varphi)\right]$.

Example 3. $\widehat{R \bigcirc R q}=R \bigcirc p_{R q}$

Definition 18. For an instance $(\mathcal{G}, T, \varphi)$ of $\mathrm{FUS}_{n+1}$, we define the instance $(\widehat{\mathcal{G}, T, \varphi})$ of $\mathrm{FUS}_{n}$ as $\left(\mathcal{G}^{\prime}, T^{\prime}, \varphi^{\prime}\right)$ by:

- If $\widehat{\mathcal{G}}=\left(\widehat{V}, \widehat{E}, \widehat{v_{I}}, \widehat{\ell}\right)$, then $\mathcal{G}^{\prime}=\left(\widehat{V}, \widehat{E}, \widehat{v_{I}}, \widehat{\ell^{\prime}}\right)$, with

- $T^{\prime}=\widehat{T}$

$\widehat{\ell}^{\prime}(\widehat{v})=\widehat{\ell}(\widehat{v}) \cup\left\{p_{R \psi} \mid R \psi \in \mathcal{L}_{1} \cap S u b(\varphi)\right.$ and $\left.\forall u \in \widehat{v} . I, \mathcal{G}, u \models \psi\right\}$.

- $\varphi^{\prime}=\widehat{\varphi}$

From now on, for an instance $(\mathcal{G}, T, \varphi)$ of $\mathrm{FUS}_{n+1}$, we abuse notation by writing $\widehat{\mathcal{G}}=\left(\widehat{V}, \widehat{E}, \widehat{v_{I}}, \widehat{\ell}\right)$ for the modified powerset construction of Definition 18 .

Lemma 4. Take an instance $(\mathcal{G}, T, \varphi)$ of $\operatorname{FUS}_{n+1}$, and let $(\widehat{\mathcal{G}}, \widehat{T}, \widehat{\varphi})=(\widehat{\mathcal{G}, T, \varphi})$. Then for all $\pi \in$ Plays $\omega_{\omega}$ and $i \geq 0$,

$$
\pi, i \models \sim \text { iff } \widehat{\pi}, i \models \approx \widehat{\varphi}
$$

Proof. By Proposition $4, \pi, i=\sim \varphi$ iff $\widehat{\pi}, i=\lesssim \varphi$, so it only remains to show that $\widehat{\pi}, i \models \approx \varphi$ iff $\widehat{\pi}, i \models \approx \widehat{\varphi}$. We prove it by induction on $\varphi$. The cases $\varphi=p, \varphi=$ $\neg \psi, \varphi=\psi \vee \psi^{\prime}, \varphi=\bigcirc \psi, \varphi=\psi \mathcal{U} \psi^{\prime}$ are trivial. It remains to consider the case $\varphi=R \psi$, which decomposes into two subcases depending on $d_{R}(\psi)$ :

- If $d_{R}(\psi)>0$, then $\widehat{\varphi}=R \widehat{\psi}$. We then have:

$$
\begin{aligned}
\widehat{\pi}, i=\approx R \psi & \text { iff } \forall \widehat{\pi}^{\prime}, j \text { s.t. } \widehat{\pi}[0, i] \widehat{\approx} \widehat{\pi}^{\prime}[0, j], \widehat{\pi}^{\prime}, j \models \approx \psi \\
& \text { iff } \forall \widehat{\pi}^{\prime}, j \text { s.t. } \widehat{\pi}[0, i] \widehat{\approx} \widehat{\pi}^{\prime}[0, j], \widehat{\pi}^{\prime}, j \models \approx \widehat{\psi} \\
& \text { iff } \widehat{\pi}, i \models \approx R \widehat{\psi}
\end{aligned}
$$

- If $d_{R}(\psi)=0$, that is $\psi \in$ LTL, then $\widehat{\varphi}=p_{R \psi}$.

$$
\begin{aligned}
& \widehat{\pi}, i \models \approx R \psi \text { iff } \mathcal{G}, u \models \psi \text { for all } u \in \widehat{\pi}[i] . I \\
& \text { iff } p_{R \psi} \in \widehat{\ell}(\widehat{\pi}[i]) \\
& \text { iff } \widehat{\pi}, i=\approx p_{R \psi}
\end{aligned}
$$


We can now achieve the proof of Proposition 5. Take an instance $(\mathcal{G}, T, \varphi)$ of $\mathrm{FUS}_{n+1}$. We show that $(\widehat{\mathcal{G}}, \widehat{T}, \widehat{\varphi})=(\widehat{\mathcal{G}, T, \varphi})$ is a good candidate. Notice that the natural bijection between Plays $_{*}$ and $\widehat{\text { Plays }}_{*}$ induces a bijection between strategies $\sigma$ in $\mathcal{G}$ and strategies $\widehat{\sigma}$ in $\widehat{\mathcal{G}}$, such that for every strategy $\sigma$ in $\mathcal{G}$, if we note $\widehat{\operatorname{Out}(\sigma}):=$ $\{\widehat{\pi} \mid \pi \in \operatorname{Out}(\sigma)\}$, then $\operatorname{Out}(\widehat{\sigma})=\widehat{\operatorname{Out}(\sigma})$.

Let $\sigma$ be $([T], \varphi)$-fully-uniform in $\mathcal{G}$. If $\widehat{\pi} \in \operatorname{Out}(\widehat{\sigma})$, then $\widehat{\pi} \in \widehat{\operatorname{Out}(\sigma})$, hence $\pi \in$ $\operatorname{Out}(\sigma)$. Because $\sigma$ is $([T], \varphi)$-fully-uniform, $\pi, 0 \models \sim \varphi$. By Lemma 4 we conclude that $\widehat{\pi}, 0 \models \approx \widehat{\varphi}$, which means that $\widehat{\sigma}$ is $([\widehat{T}], \widehat{\varphi})$-fully-uniform in $\widehat{\mathcal{G}}$. Since $d_{R}(\widehat{\varphi})=$ $d_{R}(\varphi)-1=n,(\widehat{\mathcal{G}, T, \varphi}) \in \mathrm{FUS}_{n}$.

Assume $(\widehat{\mathcal{G}, T, \varphi}) \in \mathrm{FUS}_{n}$, that is there exists a strategy $\widehat{\sigma}$ which is $([\widehat{T}], \widehat{\varphi})$-fullyuniform in $\widehat{\mathcal{G}}$. Any play $\pi \in \operatorname{Out}(\sigma)$ is uniquely associated to a play $\widehat{\pi} \in \widehat{\operatorname{Out}(\sigma})=$ $\operatorname{Out}(\widehat{\sigma})$, which by assumption satisfies $\widehat{\pi}, 0=\approx \widehat{\varphi}$. By Lemma $4, \pi, 0=\sim \varphi$, which shows that $\sigma$ is $([T], \varphi)$-fully-uniform in $\mathcal{G}$.

This achieves the proof of the first point of Proposition 5. For the second point, recall Definition 14 that gives $|\widehat{\mathcal{G}}|=O\left(|\mathcal{G}| \times 2^{|T|} \times 2^{|T||\mathcal{G}|}\right)$, so that $|\widehat{\mathcal{G}}|=$ $O\left(2^{(|\mathcal{G}|+|T|)^{2}}\right)$. The third point is derived from this second point and Definition 16 : $|\widehat{T}|=O(|\widehat{\mathcal{G}}| \times|T| \times|\widehat{\mathcal{G}}|)=O\left(2^{(|\mathcal{G}|+|T|)^{2}} \times|T| \times 2^{(|\mathcal{G}|+|T|)^{2}}\right)=O\left(2^{O(|\mathcal{G}|+|T|)^{2}}\right)$. Finally, the fourth point stating that $|\widehat{\varphi}|=O(|\varphi|)$ is immediate by Definition 17 .

It remains to prove that $(\widehat{\mathcal{G}, T, \varphi})$ is computed in time exponential in $|(\mathcal{G}, T, \varphi)|$. Clearly, the powerset construction (Section 5.1) and the lifting of the transducer (Section 5.2) both take exponential time in $|\mathcal{G}|+|T|$, hence in $|(\mathcal{G}, T, \varphi)|$. The marking phase in the $R$-elimination (Definition 18) involves model checking at most $|\varphi|$ LTL-formulas on each position of the original arena $\mathcal{G}$. Model checking an LTLformula in a given position requires polynomial space in $|\mathcal{G}|+|\varphi|$ (Sistla and Clarke [1985]). Since PSPaCE $\subseteq$ EXPTime, it is exponential in time. All in all, we need to model check an LTL-formula at most $|\mathcal{G}| \times|\varphi|$ times, so the whole marking phase is done in time exponential in $|(\mathcal{G}, T, \varphi)|$. We conclude that $(\widehat{\mathcal{G}, T, \varphi})$ can be computed in time exponential in $|(\mathcal{G}, T, \varphi)|$, which terminates the proof of Proposition 5.

\subsection{Complexity of $\mathrm{FUS}_{n}$}

In this subsection we describe an algorithm that decides whether an instance $(\mathcal{G}, T, \varphi)$ is in FUS, and we establish upper bounds for the $\mathrm{FUS}_{n}$ problem, for each $n \in \mathbb{N}$.

Algorithm 1 describes our decision procedure. It takes as an entry an instance $(\mathcal{G}, T, \varphi)$ of FUS, and returns true if it is a positive instance ${ }^{\mathrm{c}}$, false otherwise. To do so, starting from $\left(\mathcal{G}_{0}, T_{0}, \varphi_{0}\right)=(\mathcal{G}, T, \varphi)$, it successively applies the construction

${ }^{c}$ i.e., there exists a $([T], \varphi)$-fully-uniform strategy in $\mathcal{G}$. 
described in Subsection 5.3 to eliminate $R$ operators in $\varphi$ and to ultimately reduce the problem to solving an equivalent LTL game.

It is known that solving LTL games has a time complexity doubly-exponential in the size of the formula, and that it is actually 2ExpTIME-complete (Pnueli and Rosner [1989]). We remind that solving an LTL game $(\mathcal{G}, \varphi)$, in the automatatheoretic formulation of this problem (Vardi [1991]), can be done by the following procedure, that we will call LTLGameSolver (see Pnueli and Rosner [1989]; Alur and La Torre [2004]). First, compute a nondeterministic Büchi tree automaton that accepts trees whose branches all verify the formula. This automaton is of size exponential in $|\varphi|$. Then, by for example Safra's construction (Safra [1988]), build an equivalent deterministic Rabin automaton $\mathcal{A}_{\varphi}$ with a number of states doublyexponential in $|\varphi|$, and a number of pairs exponential in $|\varphi|$. Then, with a linear cost in $|\mathcal{G}|$, transform the arena $\mathcal{G}$ into a nondeterministic tree automaton $\mathcal{A}_{\mathcal{G}}$ that accepts all strategies of Player 1 in $\mathcal{G}$. Then, there exists a strategy whose outcomes all satisfy $\varphi$ if and only if the product Rabin automaton $\mathcal{A}_{\varphi} \times \mathcal{A}_{\mathcal{G}}$ accepts some tree. Deciding the emptiness of a Rabin tree automaton can be done time $O\left((\ell m)^{3 m}\right)$, where $\ell$ is the number of states and $m$ is the number of pairs of the Rabin automaton Rosner [1991]. Provided that for the product Rabin automaton $\mathcal{A}_{\varphi} \times \mathcal{A}_{\mathcal{G}}$ we have $\ell=|\mathcal{G}| \times 2^{2^{|\varphi|}}$ and $m=2^{|\varphi|}$, we finally obtain the following upper bound:

Proposition 6. Solving an LTL game $(\mathcal{G}, \varphi)$ takes time $|\mathcal{G}|^{2^{O(|\varphi|)}}$.

It is important to keep the size of the arena and the size of the formula apart for the moment, instead of just saying that it is doubly-exponential in the size of the entry, because in our decision procedure, the size of the iterated powerset constructions suffers a exponential blow-up, contrary to the successive formulas whose sizes remain unchanged (and even decrease since subformulas are replaced with atomic propositions).

Input: $(\mathcal{G}, T, \varphi)$

Output: true if $(\mathcal{G}, T, \varphi) \in$ FUS, false otherwise $\left(\mathcal{G}_{0}, T_{0}, \varphi_{0}\right):=(\mathcal{G}, T, \varphi)$

$k:=0$;

while $d_{R}\left(\varphi_{k}\right)>0$ do

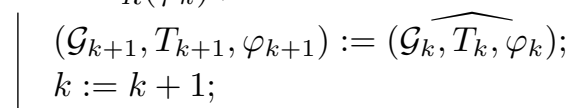

end return $L T L G a m e$ Solver $\left(\mathcal{G}_{k}, \varphi_{k}\right)$

Algorithm 1: Decision procedure for the problem FUS.

Theorem 6 as announced at the beginning of Section 5 can now be proved.

Let $n \in \mathbb{N}$, and let $(\mathcal{G}, T, \varphi)$ be an instance of $\mathrm{FUS}_{n}$. If $n=0$, the body of the while instruction is not executed, and we immediately call 
LTLGameSolver $\left(\mathcal{G}_{0}, \varphi_{0}\right)$. By Proposition 6 , this call takes time $\left|\mathcal{G}_{0}\right|^{2^{O\left(\left|\varphi_{0}\right|\right)}}$, hence it is 2-Exptime in $|(\mathcal{G}, T, \varphi)|$. We now treat the case $n>0$.

For convenience, we introduce notations for iterated exponential functions: for $\left.k, n \in \mathbb{N}, \exp ^{k}(n)=2^{2 \ldots 2^{n}}\right\} k$.

Lemma 5. For every $0 \leq k \leq n,\left|\mathcal{G}_{k}\right|=\left|T_{k}\right|=\exp ^{k}\left(O(|\mathcal{G}|+|T|)^{2}\right)$.

Proof. By induction on $k$, using Proposition 5.

If $(\mathcal{G}, T, \varphi)$ is an instance of $\mathrm{FUS}_{n}$, for $1 \leq k \leq n$, by Proposition 5 , the execution of the $k$-th loop takes time exponential in $\left|\left(\mathcal{G}_{k-1}, T_{k-1}, \varphi_{k-1}\right)\right|$. Hence by Lemma 5 , the time complexity for the $k$-th loop is $\exp ^{1}\left(\exp ^{k-1}\left(O(|\mathcal{G}|+|T|)^{2}\right)+|\varphi|\right)=$ $2^{|\varphi|} \exp ^{k}\left(O(|\mathcal{G}|+|T|)^{2}\right)$, and the execution of the whole while instruction takes time $\theta_{\text {WHILE }}$ where:

$$
\begin{aligned}
\theta_{\text {WHILE }} & =\sum_{k=1}^{n} 2^{|\varphi|} \exp ^{k}\left(O(|\mathcal{G}|+|T|)^{2}\right) \\
& =2^{|\varphi|} \exp ^{n}\left(O(|\mathcal{G}|+|T|)^{2}\right)
\end{aligned}
$$

By Proposition 6 and Lemma 5, solving the final LTL game $\left(\mathcal{G}_{n}, \varphi_{n}\right)$ takes time $\theta_{\text {LTL }}$, where:

$$
\begin{aligned}
\theta_{\mathrm{LTL}} & =\left|\mathcal{G}_{n}\right|^{2^{O\left(\left|\varphi_{n}\right|\right)}} \\
& =\exp ^{1}\left(\exp ^{n-1}\left(O(|\mathcal{G}|+|T|)^{2}\right) * 2^{O(|\varphi|)}\right)
\end{aligned}
$$

We obtain that Algorithm 1 runs in time $\theta=\theta_{\mathrm{WHILE}}+\theta_{\mathrm{LTL}}$, but since (for $n>0), \theta_{\text {WHILE }}$ is negligible compared to $\theta_{\text {LTL }}$, we obtain:

$$
\theta=\exp ^{1}\left(\exp ^{n-1}\left(O(|\mathcal{G}|+|T|)^{2}\right) * 2^{O(|\varphi|)}\right)
$$

We observe that for $n=1$, the double exponential complexity stems from the size of the formula. For $n=2$, because the size of the arena has taken two exponentials, the double exponential complexity comes both from the size of the formula and the size of the arena. Afterwards, since the arena keeps growing exponentially while the size of the formula remains the same, the complexity comes essentially from the size of the arena. This achieves the proof of Theorem 6 .

Note that the subroutine LTLGameSolver $\left(\mathcal{G}_{n}, \varphi_{n}\right)$ of Algorithm 1, based on the automata-theoretic procedure of Pnueli and Rosner [1989], does not merely decide the existence of a winning strategy, but actually builds one (if any). Recall also that forgetful strategies are sufficient for LTL games, as they are particular cases of regular games which enjoy the "Forgetful Determinacy" (Zeitman [1994]). By the natural bijection invoked in the proof of Proposition 5 between strategies in a powerset arena and strategies in the original arena, one can trace the strategy in $\mathcal{G}_{n}$ back to a $\left(\left[T_{0}\right], \varphi_{0}\right)$-fully-uniform strategy in the original game $\mathcal{G}_{0}$.

Corollary 2. Forgetful strategies are sufficient for full-uniformity properties. 


\section{Discussion}

We have investigated the concept of uniform strategies in two-player turn-based infinite-duration games, motivated by the many instances from the literature: games with imperfect information, games with epistemic condition, non-interference, diagnosis and prognosis, and Dependence Logic. Uniformity is addressed in the context of a semantic binary relation between plays of the arena, which can arise from any reason to relate plays with each others, e.g. an epistemic feature.

In order to embrace all the examples we have encountered, we were led to designing a formal language whose sentences express the very uniformity properties of strategies. Clearly, besides allowing for automated decidions procedures for the existence of uniform strategies, the language-based approach offers intuitive definitions. On the other hand, the set-theoretic one which may capture a larger class of uniformity properties, not only makes automation hopeless, but is also much less readable. The particular uniformity properties that have been addressed in the literature so far (Section 3) can now more easily be compared. Our language is an enrichment of the Linear-time Temporal logic LTL (Gabbay et al. [1980], ), hence it is interpreted over plays. The additional feature is captured by the modality $R$ which quantifies universally over related plays. Whether this quantification ranges over all plays in the arena or just over outcomes of the considered strategy yields two variants of uniform strategies, namely fully-uniform and strictly-uniform strategies.

The general procedure to decide the fully-uniform strategy problem is nonelementary. This may be the price to pay for a generic solution for arbitrarily complex uniformity properties, and we conjecture that the fully-uniform strategy problem is non-elementary hard. However, bounding the $R$-depth of the formulas gives an elementary bound complexity, which seems incidentally to be the case for all the examples of Section 3: only formulas whose $R$-depth is one are needed, so that the generic procedure has "only" a double exponential time complexity. Notice that Maubert et al. [2011], obtained a tighter (optimal) single exponential time bound for solving games with opacity condition, which corresponds to the fixed formula $\mathbf{G} \neg R p_{S}$ of $R$-depth equal to one and to a simple fixed binary relation between plays (see Section 3.2). Notice that if we fix a formula of $R$-depth 1 , the time complexity Equation (17) of our procedure collapses to a single exponential time complexity in the size of the arena and of the transducer.

Our results can be extended and commented in many respects. We give here some of them.

First, the choice we have made to rely on an enrichment of the LTL logic can be questioned - although this logic regarding properties of time is acknowledged in

many respects. We may try to extend the synthesis procedure to a much richer logic like the Linear-time $\mu$-calculus, a language extending standard linear time temporal logic with fix-point operators. But the current procedure relies on a bottom-up 
traversal of the parse-tree of the formula $\varphi$, which cannot be generalized to formulas with arbitrary fix-points. The LTL logic falls into the very particular so-called alternation-free fragment of the Linear-time $\mu$-calculus, where fix-points do not interplay. Significant progress in understanding this extended setting need being pursued.

Second, we considered a single semantic binary relation between plays. One may wonder whether the case of several relations $\sim{ }_{i}$, yielding modalities $R_{i}$ at the language level, can be investigated at the algorithmic level. We foresee a generalization of our powerset construction by synchronizing the execution of all the transducers of the relations. We however remain cautious regarding the success of this approach since closely related topics such as the decentralized diagnosis problem is known to be undecidable (Sengupta and Tripakis [2002]). Still the question is important as it would unify our setting with the Epistemic Temporal Logic (ETL) of Halpern and Vardi [1989] and bring light on the automated verification of ETL definable properties for open systems (see the module-checking problem of Kupferman and Vardi [1997]).

Additional comments are needed to fully understand the contribution, in particular regarding the recent developments of alternating-time epistemic logic (van der Hoek and Wooldridge [2003]; Jamroga and van der Hoek [2004]; Dima et al. [2010], ). The two settings are close but incomparable. With the uniform strategy concept, we aim at extending the range of (qualitative) properties of strategies by means of binary relations between plays, and at exploiting those properties to synthesize particular strategies. Instead, alternating-time epistemic logics offer a way to quantify over strategies that achieve ETL-like properties, hence they are not synthesis-oriented, and moreover, they do not handle arbitrary relations between plays. Unifying the two settings is a real challenge; we would need to design a (necessarily more complex) language that incorporates the specification of the relation(s) between plays.

\section{References}

Alur, R., Černỳ, P. and Chaudhuri, S. [2007] Model checking on trees with path equivalences. Tools and Algorithms for the Construction and Analysis of Systems, 664-678.

Alur, R., Černý, P. and Zdancewic, S. [2006] Preserving secrecy under refinement. In ICALP '06: Proceedings (Part II) of the 33rd International Colloquium on Automata, Languages and Programming, 107-118. Springer.

Alur, R., Henzinger, T. and Kupferman, O. [2002] Alternating-time temporal logic. Journal of the ACM (JACM), 49, 5, 672-713.

Alur, R. and La Torre, S. [2004] Deterministic generators and games for ltl fragments. ACM Transactions on Computational Logic (TOCL), 5, 1, 1-25.

Apt, K. and Grädel, E. [2011] Lectures in Game Theory for Computer Scientists. Cambridge University Press. 
Arnold, A., Vincent, A. and Walukiewicz, I. [2003] Games for synthesis of controllers with partial observation. Theoretical Computer Science, 1, 303, 7-34.

Van Benthem, J. [2005] The epistemic logic of if games. The Philosophy of Jaakko Hintikka, 30.

Berstel, J. [1979] Transductions and context-free languages, volume 4. Teubner Stuttgart. Berwanger, D. and Doyen, L. [2008] On the power of imperfect information. In R. Hariharan, M. Mukund and V. Vinay, editors, FSTTCS, volume 2 of LIPIcs, 73-82. Schloss Dagstuhl - Leibniz-Zentrum fuer Informatik.

Cassandras, C. and Lafortune, S. [1999] Discrete event systems- the state of the art and new directions. Applied and computational control, signals, and circuits., 1, 1-65.

Chatterjee, K., Doyen, L., Henzinger, T. and Raskin, J. [2006] Algorithms for omegaregular games with imperfect information. In Computer Science Logic, 287-302. Springer.

Dima, C., Enea, C. and Guelev, D. [2010] Model-checking an alternating-time temporal logic with knowledge, imperfect information, perfect recall and communicating coalitions. Electronic Proceedings in Theoretical Computer Science, 25.

Elgot, C. and Mezei, J. [1965] On relations defined by generalized finite automata. IBM Journal of Research and Development, 9, 1, 47-68.

Emerson, E. A. and Jutla, C. S. [1991] Tree automata, mu-calculus and determinacy (extended abstract). In FOCS, 368-377. IEEE Computer Society.

Fagin, R., Halpern, J. and Vardi, M. [1991] A model-theoretic analysis of knowledge. Journal of the ACM (JACM), 38, 2, 382-428.

Gabbay, D. M., Pnueli, A., Shelah, S. and Stavi, J. [1980] On the temporal basis of fairness. In P. W. Abrahams, R. J. Lipton and S. R. Bourne, editors, POPL, 163-173. ACM Press.

Goguen, J. and Meseguer, J. [1982] Security policies and security models. In IEEE Symposium on Security and privacy, volume 12 .

Grädel, E., Thomas, W. and Wilke, T., editors [2002] Automata, Logics, and Infinite Games: A Guide to Current Research [outcome of a Dagstuhl seminar, February 2001], volume 2500 of Lecture Notes in Computer Science. Springer.

Halpern, J. and Vardi, M. [1989] The complexity of reasoning about knowledge and time. 1. lower bounds. Journal of Computer and System Sciences, 38, 1, 195-237.

Hintikka, J. [1962] Knowledge and belief, volume 13. Cornell University Press Ithaca.

Hintikka, J. and Sandu, G. [1989] Informational independence as a semantical phenomenon. Studies in Logic and the Foundations of Mathematics, 126, 571-589.

Jamroga, W. and van der Hoek, W. [2004] Agents that know how to play. Fundamenta Informaticae, 63, 2-3, 185-219.

Kupferman, O. and Vardi, M. Y. [1997] Module checking revisited. In Computer Aided Verification, Proc. 9th Int. Conference, volume 1254 of Lecture Notes in Computer Science, 36-47. Springer-Verlag.

Ladner, R. E. and Reif, J. H. [1986] The logic of distributed protocols. In J. Y. Halpern, editor, TARK, 207-222. Morgan Kaufmann.

Lehmann, D. [1984] Knowledge, common knowledge and related puzzles (extended summary). In Proceedings of the third annual ACM symposium on Principles of distributed computing, 62-67. ACM.

Maubert, B., Pinchinat, S. and Bozzelli, L. [2011] Opacity issues in games with imperfect information. In G. D'Agostino and S. L. Torre, editors, GandALF, volume 54 of EPTCS, 87-101.

Parikh, R. and Ramanujam, R. [1985] Distributed processes and the logic of knowledge. Logics of Programs, 256-268. 
Piccione, M. and Rubinstein, A. [1997] The absent-minded driver's paradox: synthesis and responses. Games and Economic Behavior, 20, 1, 121-130.

Pinchinat, S. and Riedweg, S. [2005] A decidable class of problems for control under partial observation. Inf. Process. Lett., 95, 4, 454-460.

Pnueli, A. and Rosner, R. [1989] On the synthesis of an asynchronous reactive module. In Proc. 16th Int. Coll. on Automata, Languages and Programming, ICALP'89, Stresa, Italy, LNCS 372, 652-671. Springer-Verlag.

Reif, J. [1984] The complexity of two-player games of incomplete information. Journal of computer and system sciences, 29, 2, 274-301.

Rosner, R. [1991] Modular synthesis of reactive systems. Ann Arbor, 1050, 48106-1346.

Safra, S. [1988] On the complexity of $\omega$-automata. In Proc. 29th IEEE Symp. Foundations of Computer Science, White Plains, New York, 319-327.

Sampath, M., Sengupta, R., Lafortune, S., Sinaamohideen, K. and Teneketzis, D. [1995] Diagnosability of discrete event systems. IEEE Transactions on Automatic Control, 40, 9, 1555-1575.

Sato, J. [1977] A study of kripke style methods for some modal logic by gentzen's sequential method. Technical report, Technical report, Publication Research Institute for Mathematical Science.

Sengupta, R. and Tripakis, S. [2002] Decentralized diagnosability of regular languages is undecidable. In Decision and Control, 2002, Proceedings of the 41st IEEE Conference on, volume 1, 423-428. IEEE.

Sistla, A. P. and Clarke, E. M. [1985] The complexity of propositional linear temporal logics. Journal of the ACM, 32, 3, 733-749.

Väänänen, J. [2007] Dependence logic.

Van Benthem, J. [2001] Games in dynamic-epistemic logic. Bulletin of Economic Research, 53, 4, 219-248.

van der Hoek, W. and Wooldridge, M. [2003] Cooperation, knowledge, and time: Alternating-time temporal epistemic logic and its applications. Studia Logica, 75, $1,125-157$.

Vardi, M. [1991] Verification of concurrent programs: The automata-theoretic framework. Annals of Pure and Applied Logic, 51, 1, 79-98.

Yoo, T. and Lafortune, S. [2002] Polynomial-time verification of diagnosability of partially observed discrete-event systems. Automatic Control, IEEE Transactions on, 47, 9, 1491-1495.

Zeitman, S. [1994] Unforgettable forgetful determinacy. Journal of Logic and Computation, 4, 3, 273-283. 